\title{
THE MEAN-FIELD LIMIT FOR A REGULARIZED VLASOV-MAXWELL DYNAMICS
}

\author{
FRANÇOIS GOLSE
}

\begin{abstract}
The present work establishes the mean-field limit of a $N$-particle system towards a regularized variant of the relativistic Vlasov-Maxwell system, following the work of Braun-Hepp [Comm. in Math. Phys. 56 (1977), 101-113] and Dobrushin [Func. Anal. Appl. 13 (1979), 115-123] for the Vlasov-Poisson system. The main ingredients in the analysis of this system are (a) a kinetic formulation of the Maxwell equations in terms of a distribution of electromagnetic potential in the momentum variable, (b) a regularization procedure for which an analogue of the total energy - i.e. the kinetic energy of the particles plus the energy of the electromagnetic field - is conserved and (c) an analogue of Dobrushin's stability estimate for the Monge-Kantorovich-Rubinstein distance between two solutions of the regularized Vlasov-Poisson dynamics adapted to retarded potentials.
\end{abstract}

\section{INTRODUCTION}

Vlasov equations are kinetic equations used to describe the evolution of dilute, collisionless systems of particles coupled by some long-range interaction. For instance, in the case of a rarefied plasma, each charged particle is accelerated by the electrostatic field created by all the other particles, a situation described by the Vlasov-Poisson system [20]. A similar Vlasov-Poisson system is used in astrophysics to model the collective behavior of like massive objects coupled by Newton's gravitational force - which is attractive, at variance with the electrostatic force between charged particles with charges of the same sign.

Other types of interactions can also be considered. In the case of magnetized plasmas - e.g. plasmas in tokamak machines - each charged particle is accelerated by the Lorentz force resulting from the electromagnetic field created by all the other particles, a situation described by the Vlasov-Maxwell system [13]. There are also general

1991 Mathematics Subject Classification. 82C22, 35Q83, 35Q61 (82D10).

Key words and phrases. Mean-field limit; Vlasov-Maxwell system; LiénardWiechert potential, Monge-Kantorovich-Rubinstein distance. 
relativistic variants of the gravitational Vlasov-Poisson system, such as the Vlasov-Einstein [5] or the (model) Vlasov-Nordström systems [4].

At the time of this writing, there is no rigorous derivation of any of these kinetic equations from the corresponding $N$-body problems, in the large $N$ limit. Braun and Hepp [3] and Dobrushin [7] have proposed instead rigorous derivations of a system analogous to the Vlasov-Poisson system, replacing the Coulomb potential with a twice differentiable mollification thereof. Hauray and Jabin [10] have succeeded in treating the case of singular potentials, but their analysis does not include the Coulomb singularity yet. It should be noted that the situation is significantly better in the quantum case: the Hartree or Schrödinger-Poisson equation, that is the quantum analogue of the Vlasov-Poisson system has been derived from the quantum $N$-body problem with Coulomb potential by Erdös and Yau [9].

In the case of the Vlasov-Maxwell system, it is not even clear what the corresponding $N$-body problem should be. For instance, there is at present no satisfying description of the electromagnetic self-interaction (i.e. the action of the electromagnetic field created by a moving, pointlike charge on itself) within the theory of classical electrodynamics. Bibliographic references discussing this well-known issue can be found in the remarks following the introduction of the regularized $N$-body system (27).

The present paper proposes an analogue of the Braun-Hepp or Dobrushin theory for (a mollified version of) the Vlasov-Maxwell system. Elskens, Kiessling and Ricci [8] have recently considered a special relativistic variant of the gravitational Vlasov-Poisson system where the Poisson equation for the potential is replaced with a linear wave equation.

The problem of deriving a regularized variant of the Vlasov-Maxwell equations from a particle system is explicitly mentioned by Kiessling on p. 111 in his survey article [12]. As expected, this problem can be handled more or less along the line of [8], with however a few significant modifications, which it is the purpose of the present work to explain. As we shall see, the regularization procedure removes all the conceptual difficulties pertaining to the electromagnetic self-interaction mentioned above. The idea of considering a mollified variant of the Vlasov-Maxwell dynamics is therefore motivated by reasons other than analytical simplicity. 


\section{A scalar Formulation of the Vlasov-Maxwell System}

Regularizing the Coulomb potential is a crucial step in the meanfield limit established by Braun and Hepp [3] and Dobrushin [7]. At first sight, it is not clear that the same procedure can be applied to the Vlasov-Maxwell system, as the electromagnetic field involves both a scalar and vector potentials. Perhaps for that reason, Elskens, Kiessling and Ricci [8] chose "to purge the inhomogeneous wave equation for [the vector potential] $A$ and all terms involving (derivatives of) $A$ in the Lorentz force" and retain only the inhomogeneous wave equation for the scalar potential.

In this section, we explain how both the scalar and vector potentials in the Vlasov-Maxwell system can be expressed in terms of a single scalar potential distributed in the momentum variable. This formulation, introduced in $[1,2]$ for other purposes — viz. for studying regularity properties of the Vlasov-Maxwell system - although perhaps not absolutely necessary in the present context, greatly simplifies our arguments in the sequel.

The Vlasov-Maxwell system for a single species of particles with momentum distribution function $f(t, x, \xi)$ - meaning that $f(t, x, \xi)$ is the density of particles with momentum $\xi \in \mathbf{R}^{3}$ that are located at the position $x \in \mathbf{R}^{3}$ at time $t \geq 0$ - is written as follows:

$$
\left\{\begin{array}{l}
\partial_{t} f+v(\xi) \cdot \nabla_{x} f+(E+v(\xi) \wedge B) \cdot \nabla_{\xi} f=0 \\
\operatorname{div}_{x} B=0 \\
\partial_{t} B+\operatorname{curl}_{x} E=0 \\
\operatorname{div}_{x} E=\rho_{f} \\
\partial_{t} E-\operatorname{curl}_{x} B=-j_{f} .
\end{array}\right.
$$

We have used the notation

$$
v(\xi):=\nabla_{\xi} e(\xi)=\frac{\xi}{\sqrt{1+|\xi|^{2}}}, \quad \text { where } e(\xi):=\sqrt{1+|\xi|^{2}}
$$

and

$$
\rho_{f}(t, x):=\int_{\mathbf{R}^{3}} f(t, x, \xi) d \xi, \quad \text { while } j_{f}(t, x):=\int_{\mathbf{R}^{3}} v(\xi) f(t, x, \xi) d \xi .
$$

This system is posed in the whole Euclidian space - i.e. for all $x, \xi \in \mathbf{R}^{3}$ - and supplemented with the initial data

$$
\left.f\right|_{t=0}=f^{i n},\left.\quad E\right|_{t=0}=E^{i n},\left.\quad B\right|_{t=0}=0,
$$


where $E^{i n}$ is the electrostatic field created by the distribution of charges $f^{\text {in }}$ :

$$
E^{i n}=-\nabla_{x} \phi^{i n}, \quad \phi^{i n}=\left(-\Delta_{x}\right)^{-1} \int_{\mathbf{R}^{3}} f^{i n} d \xi .
$$

(We restrict our attention to such initial data for simplicity; treating the case of general initial data

$$
\left.f\right|_{t=0}=f^{i n},\left.\quad E\right|_{t=0}=E^{i n},\left.\quad B\right|_{t=0}=B^{i n},
$$

with the compatibility conditions

$$
\operatorname{div}_{x} E^{i n}=\int_{\mathbf{R}^{3}} f^{i n} d \xi, \quad \operatorname{div}_{x} B^{i n}=0
$$

is not significantly more complicated, as it only involves one extra linear, homogeneous wave equation.)

Solve for $\phi_{0}$ the Cauchy problem for the linear wave equation

$$
\left\{\begin{array}{l}
\square_{t, x} \phi_{0}=0, \\
\left.\phi_{0}\right|_{t=0}=\phi^{i n}=\left(-\Delta_{x}\right)^{-1} \int_{\mathbf{R}^{3}} f^{i n} d \xi, \\
\left.\partial_{t} \phi_{0}\right|_{t=0}=0 .
\end{array}\right.
$$

The scalar formulation of (1) is as follows. Consider the coupled system with unknowns $f \equiv f(t, x, \xi) \geq 0$ and $u \equiv u(t, x, \xi) \in \mathbf{R}$ :

$$
\left\{\begin{array}{l}
\partial_{t} f+v(\xi) \cdot \nabla_{x} f+(E+v(\xi) \wedge B) \cdot \nabla_{\xi} f=0, \\
\square_{t, x} u=f, \\
\phi=\phi_{0}+\int_{\mathbf{R}^{3}} u d \xi, \quad A=\int_{\mathbf{R}^{3}} v(\xi) u d \xi, \\
B=\operatorname{curl}_{x} A, \quad E=-\partial_{t} A-\nabla_{x} \phi,
\end{array}\right.
$$

with initial data

$$
\left.f\right|_{t=0}=f^{i n},\left.\quad u\right|_{t=0}=\left.\partial_{t} u\right|_{t=0}=0 .
$$

Physically, $u(t, x, \xi)$ is the instantaneous Liénard-Wiechert potential created by a particle with momentum $\xi$ at the position $x$, distributed under $f(t, x, \xi)$.

Proposition 2.1. Let $f \in C_{c}^{\infty}\left([0, T] \times \mathbf{R}^{3} \times \mathbf{R}^{3}\right)$, and let $\phi_{0}$ be the solution of (4). If $(f, u)$ satisfies (5) with initial data (6), then the 
electromagnetic potential $(\phi, A)$ defined as in (5) is smooth and satisfies the Lorentz gauge

$$
\partial_{t} \phi+\operatorname{div}_{x} A=0
$$

while $(f, E, B)$, with the electromagnetic field $(E, B)$ as in (5), satisfies (1) with initial condition (2)-(3).

We shall use systematically the following elements of notation: $C_{c}(X)$ (resp. $\left.C_{c}^{k}(X), C_{c}^{\infty}(X)\right)$ designates the set of continuous (resp. $C^{k}, C^{\infty}$ ) functions with compact support included in $X$. Likewise, $C_{b}(X)$ designates the set of bounded continuous functions defined on $X$, while $C_{b}^{k}(X)$ designates the set of $C^{k}$ functions defined on $X$ whose derivatives of order $\leq k$ are bouned on $X$. Finally, $C_{b}^{\infty}(X)$ designates the class of $C^{\infty}$ defined on $X$ whose derivatives of all orders are bounded on $X$.

Proof. Since $f^{i n} \in C_{c}^{\infty}\left(\mathbf{R}^{3} \times \mathbf{R}^{3}\right)$, the initial electrostatic potential

$$
\phi^{i n}=\left(-\Delta_{x}\right)^{-1} \rho_{f^{i n}} \in C_{b}^{\infty}\left(\mathbf{R}^{3}\right)
$$

so that the solution $\phi_{0}$ of the Cauchy problem (4) is in $C_{b}^{\infty}\left([0, T] \times \mathbf{R}^{3}\right)$ for each $T>0$.

Since $f \in C_{c}^{\infty}\left([0, T] \times \mathbf{R}^{3} \times \mathbf{R}^{3}\right)$, the finite speed of propagation and the regularity theory for solutions of the wave equation implies that $u \in C_{c}^{\infty}\left([0, T] \times \mathbf{R}^{3} \times \mathbf{R}^{3}\right)$.

Therefore the scalar potential $\phi \in C_{b}^{\infty}\left([0, T] \times \mathbf{R}^{3}\right)$ and the vector potential $A \in C_{c}^{\infty}\left([0, T] \times \mathbf{R}^{3} ; \mathbf{R}^{3}\right)$, so that, in view of the last equalities in (5), the electric field $E \in C_{b}^{\infty}\left([0, T] \times \mathbf{R}^{3} ; \mathbf{R}^{3}\right)$ while the magnetic field $B \in C_{c}^{\infty}\left([0, T] \times \mathbf{R}^{3} ; \mathbf{R}^{3}\right)$ and

$$
\left\{\begin{array}{l}
\operatorname{div}_{x} B=0 \\
\partial_{t} B+\operatorname{curl}_{x} E=0
\end{array}\right.
$$

Besides, $\rho_{f}$ and $j_{f}$ belong to $C_{c}^{\infty}\left([0, T] \times \mathbf{R}^{3}\right)$ and $C_{c}^{\infty}\left([0, T] \times \mathbf{R}^{3} ; \mathbf{R}^{3}\right)$ respectively, and since $v(\xi)=\nabla_{\xi} e(\xi)$, one has $\operatorname{div}_{\xi}(v(\xi) \wedge B(t, x))=$ $B(t, x) \cdot \operatorname{curl}_{\xi} v(\xi)=0$ so that

$$
\begin{aligned}
\partial_{t} \rho_{f}+\operatorname{div}_{x} j_{f} & =\int_{\mathbf{R}^{3}}\left(\partial_{t} f+v(\xi) \cdot \nabla_{x} f\right)(t, x, \xi) d \xi \\
& =\int_{\mathbf{R}^{3}} \operatorname{div}_{\xi}(-(E+v \wedge B) f)(t, x, \xi) d \xi=0 .
\end{aligned}
$$


Let us verify that $(\phi, A)$ satisfies the Lorentz gauge condition. Setting $g:=\partial_{t} \phi+\operatorname{div}_{x} A \in C_{b}^{\infty}\left([0, T] \times \mathbf{R}^{3}\right)$, one has

$$
\begin{aligned}
\square_{t, x} g & =\square_{t, x} \partial_{t} \phi_{0}+\square_{t, x} \int_{\mathbf{R}^{3}}\left(\partial_{t} u+\operatorname{div}_{x}(v(\xi) u)\right) d \xi \\
& =\partial_{t} \rho_{f}+\operatorname{div}_{x} j_{f}=0,
\end{aligned}
$$

together with the initial conditions

$$
\left.g\right|_{t=0}=\left.\partial_{t} \phi_{0}\right|_{t=0}+\int_{\mathbf{R}^{3}}\left(\left.\partial_{t} u\right|_{t=0}+\operatorname{div}_{x}\left(\left.v(\xi) u\right|_{t=0}\right)\right) d \xi=0
$$

and

$$
\begin{aligned}
\left.\partial_{t} g\right|_{t=0} & =\left.\partial_{t}^{2} \phi\right|_{t=0}+\left.\operatorname{div}_{x} \partial_{t} A\right|_{t=0} \\
& =\left.\square_{t, x} \phi_{0}\right|_{t=0}+\left.\Delta_{x} \phi\right|_{t=0}+\left.\int_{\mathbf{R}^{3}} \partial_{t}^{2} u\right|_{t=0} d \xi+\left.\int_{\mathbf{R}^{3}} v(\xi) \cdot \nabla_{x} \partial_{t} u\right|_{t=0} d \xi \\
& =\Delta_{x} \phi^{i n}+\left.\int_{\mathbf{R}^{3}} \square_{t, x} u\right|_{t=0} d \xi+\left.\int_{\mathbf{R}^{3}} \Delta_{x} u\right|_{t=0} d \xi \\
& =\Delta_{x} \phi^{i n}+\int_{\mathbf{R}^{3}} f^{i n} d \xi=0 .
\end{aligned}
$$

By the uniqueness property for the wave equation, one concludes that $g=0$, which is the Lorentz gauge condition.

It remains to verify that the electromagnetic field satisfies the Gauss and Maxwell-Ampère equations: by the Lorentz gauge condition,

$$
\begin{aligned}
\operatorname{div}_{x} E & =\square_{t, x} \phi-\partial_{t}\left(\partial_{t} \phi+\operatorname{div}_{x} A\right) \\
& =\square_{t, x} \phi_{0}+\square_{t, x} \int_{\mathbf{R}^{3}} u d \xi=\int_{\mathbf{R}^{3}} f d \xi=\rho_{f}
\end{aligned}
$$

while

$$
\begin{aligned}
\partial_{t} E-\operatorname{curl}_{x} B & =-\square_{t, x} A-\nabla_{x}\left(\partial_{t} \phi+\operatorname{div}_{x} A\right) \\
& =-\square_{t, x} \int_{\mathbf{R}^{3}} v(\xi) u d \xi=-\int_{\mathbf{R}^{3}} v(\xi) f d \xi=-j_{f} .
\end{aligned}
$$

Therefore $(f, E, B)$ is a solution of the Vlasov-Maxwell system (1), and

$$
\begin{aligned}
\left.E\right|_{t=0}=-\left.\int_{\mathbf{R}^{3}} v(\xi) \partial_{t} u\right|_{t=0} d \xi-\left.\nabla_{x} \int_{\mathbf{R}^{3}} u\right|_{t=0} d \xi-\left.\nabla_{x} \phi_{0}\right|_{t=0} \\
=-\nabla_{x}\left(-\Delta_{x}\right)^{-1} \int_{\mathbf{R}^{3}} f^{i n} d \xi=E^{i n}
\end{aligned}
$$

while

$$
\left.B\right|_{t=0}=\left.\operatorname{curl}_{x} \int_{\mathbf{R}^{3}} v(\xi) u\right|_{t=0} d \xi=0
$$

so that $(E, B)$ also satisfies the initial condition (2). 
With this formulation of the Vlasov-Maxwell system, the analogue of the Coulomb potential in the Vlasov-Poisson system becomes fairly obvious. Let $Y$ be the forward fundamental solution of the d'Alembert operator, i.e. the only $Y \in \mathcal{D}^{\prime}\left(\mathbf{R} \times \mathbf{R}^{3}\right)$ satisfying

$$
\left\{\begin{array}{l}
\square_{t, x} Y=\delta_{(t, x)=(0,0)} \\
\operatorname{supp}(Y) \subset \mathbf{R}_{+} \times \mathbf{R}^{3}
\end{array}\right.
$$

We recall that, in space dimension 3 , the distribution $Y$ is given by the formula

$$
Y=\frac{\mathbf{1}_{t>0}}{4 \pi t} \delta(|x|-t),
$$

where the notation $\delta(|x|-t)$ is understood as the surface measure on the sphere of radius $t>0$ centered at the origin. Then, the function $u$ in (5) is given in terms of $f$ by the formula

$$
u(\cdot, \cdot, \xi)=Y \star_{t, x}\left(\mathbf{1}_{t>0} f(\cdot, \cdot, \xi)\right), \quad \xi \in \mathbf{R}^{3}
$$

where $\star_{t, x}$ denotes convolution in the variables $(t, x)$, while the scalar potential $\phi_{0}$ is given in terms of $f^{i n}$ by

$$
\phi_{0}(t, \cdot)=\partial_{t} Y(t, \cdot) \star_{x} G \star_{x} \int_{\mathbf{R}^{3}} f^{i n}(\cdot, \xi) d \xi, \quad t>0,
$$

where

$$
G(z)=\frac{1}{4 \pi|z|}, \quad z \in \mathbf{R}^{3}
$$

is the fundamental solution of $-\Delta_{x}$.

Therefore, the Lorentz force field $F:=E+v \wedge B$ in the VlasovMaxwell system is given in terms of the particle distribution function $f$ by the formula

$$
\begin{aligned}
F[f]= & -\int_{\mathbf{R}^{3}} \nabla_{x} \partial_{t} Y(t, \cdot) \star_{x} G \star_{x} f(0, \cdot, \eta) d \eta \\
& -\int_{\mathbf{R}^{3}}\left(\nabla_{x}+v(\eta) \partial_{t}\right) Y \star_{t, x} f(\cdot, \cdot, \eta) d \eta \\
& -\int_{\mathbf{R}^{3}} v(\xi) \wedge\left(v(\eta) \wedge \nabla_{x} Y \star_{t, x} f(\cdot, \cdot, \eta)\right) d \eta,
\end{aligned}
$$

to be compared with the formula

$$
F_{\text {Poisson }}[f]=-\int_{\mathbf{R}^{3}} \nabla_{x} G \star_{x} f(t, \cdot, \eta) d \eta
$$

in the case of the Vlasov-Poisson system. 
In the Vlasov-Poisson case, the $N$-particle dynamics considered by Hauray and Jabin (see for instance formula (1.1)-(1.2) in [10]) is the differential system

$$
\ddot{x}_{i}(t)=-\frac{1}{N-1} \sum_{\substack{j=1 \\ j \neq i}}^{N} \nabla_{x} G\left(x_{i}(t)-x_{j}(t)\right), \quad 1 \leq i \leq N
$$

By analogy, in the Vlasov-Maxwell case, one should consider the system

$$
\left\{\begin{array}{l}
\dot{x}_{i}(t)=v\left(\xi_{i}(t)\right) \\
\dot{\xi}_{i}(t)=-\frac{1}{N-1} \sum_{\substack{j=1 \\
j \neq i}}^{N} \nabla_{x} \partial_{t} Y(t, \cdot) \star_{x} G\left(x_{i}(0)-x_{j}(0)\right) \\
-\frac{1}{N-1} \sum_{\substack{j=1 \\
j \neq i}}^{N} \int_{0}^{t}\left(\nabla_{x}+v\left(\xi_{j}(s)\right) \partial_{t}\right) Y\left(t-s, x_{i}(t)-x_{j}(s)\right) d s \\
-\frac{1}{N-1} \sum_{\substack{j=1 \\
j \neq i}}^{N} \int_{0}^{t} v\left(\xi_{i}(t)\right) \wedge\left(v\left(\xi_{j}(s)\right) \wedge \nabla_{x} Y\left(t-s, x_{i}(t)-x_{j}(s)\right)\right) d s, \\
\quad i=1, \ldots, N .
\end{array}\right.
$$

There is an obvious difficulty in (12): since $\nabla_{x} G$ is singular at the origin, the right-hand side of the equation for $\dot{x}_{i}$ is not defined whenever $x_{i}(t)=x_{j}(t)$ for some $j \neq i$. More seriously, in (13), the second and third terms in the r.h.s. of the equation for $\xi_{i}$ are not a priori well defined quantities because $Y$ is a measure and not a $\left(C^{1}\right)$ function. Yet this difficulty disappears in the reference [3], where the Green function $G$ is replaced with a regularization thereof. Likewise, we shall replace $Y$ with some regularized variant thereof, which removes the difficulty mentioned above.

Notice that, while (12) is a system of $N$ coupled ordinary differential equations, (13) is a system of $N$ coupled integro-differential equations - more specifically, delay differential equations. The same is true of the dynamical system (7)-(9) considered in [8], and this is a consequence of the retarded potential formula for the solution of the Maxwell system, or, equivalently, of Kirchhoff's formula (9) for the solution of the wave equation. 


\section{A Regularization of the Vlasov-Maxwell System}

Any regularization of $Y$ would transform (13) into a well-posed system. Yet, classical solutions of the original Vlasov-Maxwell system satisfy some conservation laws, which one might wish to preserve by the regularization procedure.

Since $v=\nabla_{\xi} e$,

$$
\operatorname{div}_{x, \xi}(v(\xi), E+v \wedge B)=\operatorname{div}_{\xi}(v \wedge B)=B \cdot \operatorname{curl}_{\xi} v=0
$$

so that the characteristic field of the transport equation in (1) preserves the phase space Lebesgue measure $d x d \xi$. Hence, if $(f, E, B)$ is a classical solution of (1) on the time interval $[0, T]$, then, for all $p \in[1,+\infty]$, one has

$$
\|f(t, \cdot, \cdot)\|_{L^{p}\left(\mathbf{R}^{3} \times \mathbf{R}^{3}\right)}=\left\|f^{i n}(\cdot, \cdot)\right\|_{L^{p}\left(\mathbf{R}^{3} \times \mathbf{R}^{3}\right)}
$$

for each $t \in[0, T]$. This obviously remains true if the electromagnetic field $(E, B)$ is replaced in the transport equation governing $f$ with any regularization thereof in the $t, x$ variables.

Moreover, if $(f, E, B)$ is a classical solution of $(1)$ on the time interval $[0, T]$ with, for instance, $f \in C_{c}^{1}\left([0, T] \times \mathbf{R}^{3} \times \mathbf{R}^{3}\right)$ while the electromagnetic field $E, B \in C_{b}^{1}\left([0, T] \times \mathbf{R}^{3} ; \mathbf{R}^{3}\right) \cap C\left([0, T] ; L^{2}\left(\mathbf{R}^{3} ; \mathbf{R}^{3}\right)\right)$, then

$$
\iint_{\mathbf{R}^{3} \times \mathbf{R}^{3}} e(\xi) f(t, x, \xi) d x d \xi+\frac{1}{2} \int_{\mathbf{R}^{3}}\left(|E|^{2}+|B|^{2}\right)(t, x) d x=\text { Const. }
$$

which is the conservation of energy (the first integral being the kinetic energy of the particles while the second is the electromagnetic energy). Conservation of energy is not expected to be preserved by all regularizations of the electromagnetic field.

There is however one clever regularization procedure for the VlasovMaxwell system that preserves a quantity analogous to the energy and which converges to the energy (16) as the regularization is removed. This regularization of the Vlasov-Maxwell system, proposed by Rein in [16], is recalled below for the reader's convenience ${ }^{1}$.

Let $\chi \in C_{c}^{\infty}\left(\mathbf{R}^{3}\right)$ satisfy

$$
\chi(x)=\chi(-x) \geq 0, \quad \operatorname{supp}(\chi) \subset B(0,1), \quad \int_{\mathbf{R}^{3}} \chi(x) d x=1,
$$

and define the regularizing sequence

$$
\chi_{\epsilon}(x)=\frac{1}{\epsilon^{3}} \chi\left(\frac{x}{\epsilon}\right)
$$

\footnotetext{
${ }^{1}$ Prof. Rein kindly informed the author that this regularization procedure had been introduced earlier in this context by E. Horst in his Habilitationsschrift.
} 
for each $\epsilon>0$. Consider then, for each $\epsilon>0$, the following regularized variant of (5) with unknown $\left(f_{\epsilon}, u_{\epsilon}\right)$ :

$$
\left\{\begin{array}{l}
\partial_{t} f_{\epsilon}+v(\xi) \cdot \nabla_{x} f_{\epsilon}+\left(E_{\epsilon}+v(\xi) \wedge B_{\epsilon}\right) \cdot \nabla_{\xi} f_{\epsilon}=0, \\
\square_{t, x} u_{\epsilon}=\chi_{\epsilon} \star_{x} \chi_{\epsilon} \star_{x} f_{\epsilon}, \\
\phi_{\epsilon}=\chi_{\epsilon} \star_{x} \chi_{\epsilon} \star_{x} \phi_{0}+\int_{\mathbf{R}^{3}} u_{\epsilon} d \xi, \quad A_{\epsilon}=\int_{\mathbf{R}^{3}} v(\xi) u_{\epsilon} d \xi, \\
B_{\epsilon}=\operatorname{curl}_{x} A_{\epsilon}, \quad E_{\epsilon}=-\partial_{t} A_{\epsilon}-\nabla_{x} \phi_{\epsilon},
\end{array}\right.
$$

where $\phi_{0}$ is defined in as (4), and with the same initial data as for (5):

$$
\left.f_{\epsilon}\right|_{t=0}=f^{i n},\left.\quad u_{\epsilon}\right|_{t=0}=\left.\partial_{t} u_{\epsilon}\right|_{t=0}=0 .
$$

Along with the electromagnetic field $\left(E_{\epsilon}, B_{\epsilon}\right)$ of the regularized system $(17)$, consider $\left(\tilde{E}_{\epsilon}, \tilde{B}_{\epsilon}\right)$ defined as the solution of the Maxwell system

$$
\left\{\begin{array}{l}
\operatorname{div}_{x} \tilde{B}_{\epsilon}=0 \\
\partial_{t} \tilde{B}_{\epsilon}+\operatorname{curl}_{x} \tilde{E}_{\epsilon}=0 \\
\operatorname{div}_{x} \tilde{E}_{\epsilon}=\chi_{\epsilon} \star_{x} \rho_{f_{\epsilon}} \\
\partial_{t} \tilde{E}_{\epsilon}-\operatorname{curl}_{x} \tilde{B}_{\epsilon}=-\chi_{\epsilon} \star_{x} j_{f_{\epsilon}}
\end{array}\right.
$$

with initial data

$$
\left.\tilde{E}_{\epsilon}\right|_{t=0}=\chi_{\epsilon} \star_{x} E^{i n},\left.\quad \tilde{B}_{\epsilon}\right|_{t=0}=0 .
$$

Proposition 3.1 (Rein [16]). Let $\epsilon>0$ and $f^{i n} \in L^{\infty}\left(\mathbf{R}^{3} \times \mathbf{R}^{3}\right)$ be such that

$$
f^{\text {in }} \geq 0 \text { a.e. on } \mathbf{R}^{3} \times \mathbf{R}^{3} \text { and } \operatorname{supp}\left(f^{i n}\right) \text { is compact. }
$$

Then the regularized system (17) has a unique weak solution $\left(f_{\epsilon}, u_{\epsilon}\right)$ defined for all positive times and satisfying $u_{\epsilon} \in C_{c}^{\infty}\left([0, T] \times \mathbf{R}^{3} \times \mathbf{R}^{3}\right)$ for each $T>0$, while

$$
\begin{aligned}
& f_{\epsilon}(t, x, \xi) \geq 0 \quad \text { for a.e. }(t, x, \xi) \in \mathbf{R}_{+} \times \mathbf{R}^{3} \times \mathbf{R}^{3}, \\
& \left\|f_{\epsilon}(t, \cdot, \cdot)\right\|_{L^{p}\left(\mathbf{R}^{3} \times \mathbf{R}^{3}\right)}=\text { Const. for all } p \in[1,+\infty] \text {, and } \\
& \iint_{\mathbf{R}^{3} \times \mathbf{R}^{3}} e(\xi) f_{\epsilon}(t, x, \xi) d x d \xi+\frac{1}{2} \int_{\mathbf{R}^{3}}\left(\left|\tilde{E}_{\epsilon}\right|^{2}+\left|\tilde{B}_{\epsilon}\right|^{2}\right)(t, x) d x=\text { Const. }
\end{aligned}
$$

for each $t \geq 0$. 
Proof. The same computation as in the proof of Proposition 2.1 shows that $\left(f_{\epsilon}, E_{\epsilon}, B_{\epsilon}\right)$ satisfies

$$
\left\{\begin{array}{l}
\partial_{t} f_{\epsilon}+v(\xi) \cdot \nabla_{x} f_{\epsilon}+\left(E_{\epsilon}+v(\xi) \wedge B_{\epsilon}\right) \cdot \nabla_{\xi} f_{\epsilon}=0 \\
\operatorname{div}_{x} B_{\epsilon}=0 \\
\partial_{t} B_{\epsilon}+\operatorname{curl}_{x} E_{\epsilon}=0 \\
\operatorname{div}_{x} E_{\epsilon}=\chi_{\epsilon} \star_{x} \chi_{\epsilon} \star_{x} \rho_{f_{\epsilon}} \\
\partial_{t} E_{\epsilon}-\operatorname{curl}_{x} B_{\epsilon}=-\chi_{\epsilon} \star_{x} \chi_{\epsilon} \star_{x} j_{f_{\epsilon}}
\end{array}\right.
$$

with initial data

$$
\left.f_{\epsilon}\right|_{t=0}=f^{i n},\left.\quad E_{\epsilon}\right|_{t=0}=\chi_{\epsilon} \star_{x} \chi_{\epsilon} \star_{x} E^{i n},\left.\quad B_{\epsilon}\right|_{t=0}=0,
$$

where $E^{\text {in }}$ is defined as in (3).

This is precisely the regularized Vlasov-Maxwell system studied by Rein in [16], to which we refer for the existence of a solution.

That $f_{\epsilon} \geq 0$ a.e. on $\mathbf{R}_{+} \times \mathbf{R}^{3} \times \mathbf{R}^{3}$ and $t \mapsto\left\|f_{\epsilon}(t, \cdot, \cdot)\right\|_{L^{p}\left(\mathbf{R}^{3} \times \mathbf{R}^{3}\right)}$ is constant on $\mathbf{R}_{+}$follows from the method of characteristics for the transport equation satisfied by $f_{\epsilon}$ since $E_{\epsilon}$ and $B_{\epsilon}$ are smooth with bounded derivatives of all orders on $\mathbf{R}_{+} \times \mathbf{R}^{3} \times \mathbf{R}^{3}$ and the vector field

$$
(x, \xi) \mapsto\left(v(\xi), E_{\epsilon}(t, x)+v(\xi) \wedge B_{\epsilon}(t, x)\right)
$$

is divergence free, while $f^{i n} \geq 0$ a.e. on $\mathbf{R}^{3} \times \mathbf{R}^{3}$ and $f^{i n} \in L^{p}\left(\mathbf{R}^{3} \times \mathbf{R}^{3}\right)$ for each $p \in[1,+\infty]$.

We briefly recall from [16] the elegant argument leading to the conservation of the (pseudo-)energy for (21), which is the reason for the specific regularization procedure chosen in (21). For simplicity, we assume that $f^{\text {in }} \in C_{c}^{1}\left(\mathbf{R}^{3} \times \mathbf{R}^{3}\right)$, so that, by the method of characteristics, $f_{\epsilon} \in C_{c}^{1}\left([0, T] \times \mathbf{R}^{3} \times \mathbf{R}^{3}\right)$ for each $T>0$. Then

$$
\frac{d}{d t} \iint_{\mathbf{R}^{3} \times \mathbf{R}^{3}} e(\xi) f_{\epsilon}(t, x, \xi) d x d \xi=\int_{\mathbf{R}^{3}} E_{\epsilon} \cdot j_{f_{\epsilon}}(t, x) d x .
$$

By uniqueness of the solution of the Maxwell system, $E_{\epsilon}=\chi_{\epsilon} \star_{x} \tilde{E}_{\epsilon}$, so that

$$
\begin{aligned}
\int_{\mathbf{R}^{3}} E_{\epsilon} \cdot j_{f_{\epsilon}}(t, x) d x & =\int_{\mathbf{R}^{3}}\left(\chi_{\epsilon} \star_{x} \tilde{E}_{\epsilon}\right) \cdot j_{f_{\epsilon}}(t, x) d x \\
& =\int_{\mathbf{R}^{3}} \tilde{E}_{\epsilon} \cdot\left(\chi_{\epsilon} \star_{x} j_{f_{\epsilon}}\right)(t, x) d x
\end{aligned}
$$

where the last equality follows from the fact that $\chi_{\epsilon}$ is even. On the other hand, the classical computation leading to the conservation of 
energy in the Maxwell system (19) is as follows:

$$
\begin{aligned}
\frac{d}{d t} \int_{\mathbf{R}^{3}} \frac{1}{2}\left(\left|\tilde{E}_{\epsilon}\right|^{2}+\left|\tilde{B}_{\epsilon}\right|^{2}\right)(t, x) d x \\
\quad=\int_{\mathbf{R}^{3}}\left(\tilde{E}_{\epsilon} \cdot \partial_{t} \tilde{E}_{\epsilon}+\tilde{B}_{\epsilon} \cdot \partial_{t} \tilde{B}_{\epsilon}\right)(t, x) d x \\
\quad=-\int_{\mathbf{R}^{3}} \tilde{E}_{\epsilon} \cdot\left(\chi_{\epsilon} \star_{x} j_{f_{\epsilon}}\right)(t, x) d x \\
\quad+\int_{\mathbf{R}^{3}}\left(\tilde{E}_{\epsilon} \cdot \operatorname{curl}_{x} \tilde{B}_{\epsilon}-\tilde{B}_{\epsilon} \cdot \operatorname{curl}_{x} \tilde{E}_{\epsilon}\right)(t, x) d x
\end{aligned}
$$

Now the last integral above vanishes since

$$
\tilde{E}_{\epsilon} \cdot \operatorname{curl}_{x} \tilde{B}_{\epsilon}-\tilde{B}_{\epsilon} \cdot \operatorname{curl}_{x} \tilde{E}_{\epsilon}=-\operatorname{div}_{x}\left(E_{\epsilon} \wedge B_{\epsilon}\right)
$$

and $B_{\epsilon}(t, \cdot)$ has compact support for each $t \geq 0$. Combining (23) and (24) give the announced conservation of (pseudo-)energy for (21), and therefore for (17).

At this point, we introduce the regularized $N$-particle dynamics for the Vlasov-Maxwell system. Define

$$
Y_{\epsilon}=\chi_{\epsilon} \star_{x} \chi_{\epsilon} \star_{x} Y
$$

In other words, $Y_{\epsilon}$ is the solution of

$$
\left\{\begin{array}{l}
\square_{t, x} Y_{\epsilon}=\delta_{t=0} \otimes\left(\chi_{\epsilon} \star_{x} \chi_{\epsilon}\right), \quad \text { in } \mathcal{D}^{\prime}\left(\mathbf{R} \times \mathbf{R}^{3}\right) \\
\operatorname{supp}\left(Y_{\epsilon}\right) \subset \mathbf{R}_{+} \times \mathbf{R}^{3}
\end{array}\right.
$$

or, equivalently, $\left.Y_{\epsilon}\right|_{\mathbf{R}_{+} \times \mathbf{R}^{3}}$ is the solution of the Cauchy problem

$$
\left\{\begin{array}{l}
\square_{t, x} Y_{\epsilon}=0, \quad x \in \mathbf{R}^{3}, t>0 \\
\left.Y_{\epsilon}\right|_{t=0}=0, \\
\left.\partial_{t} Y_{\epsilon}\right|_{t=0}=\chi_{\epsilon} \star_{x} \chi_{\epsilon} .
\end{array}\right.
$$

The classical theory of regularity and the finite speed of propagation for solutions of the wave equation imply that, for each $\epsilon>0$,

$$
Y_{\epsilon} \in C^{\infty}\left(\mathbf{R}_{+} \times \mathbf{R}^{3}\right) \text { and } \operatorname{supp}\left(Y_{\epsilon}(t, \cdot)\right) \subset B(0, t+2 \epsilon) .
$$


Next we consider the system of (delay) differential equations $(27)$

$$
\left\{\begin{array}{l}
\dot{x}_{i}(t)=v\left(\xi_{i}(t)\right) \\
\dot{\xi}_{i}(t)=-\frac{1}{N} \sum_{j=1}^{N} \nabla_{x} \partial_{t} Y_{\epsilon}(t, \cdot) \star_{x} G\left(x_{i}(0)-x_{j}(0)\right) \\
-\frac{1}{N} \sum_{j=1}^{N} \int_{0}^{t}\left(\nabla_{x}+v\left(\xi_{j}(s)\right) \partial_{t}\right) Y_{\epsilon}\left(t-s, x_{i}(t)-x_{j}(s)\right) d s \\
-\frac{1}{N} \sum_{j=1}^{N} \int_{0}^{t} v\left(\xi_{i}(t)\right) \wedge\left(v\left(\xi_{j}(s)\right) \wedge \nabla_{x} Y_{\epsilon}\left(t-s, x_{i}(t)-x_{j}(s)\right)\right) d s . \\
\quad i=1, \ldots, N .
\end{array}\right.
$$

Like (13), this is a system of $N$ coupled delay differential equations. However, unlike in the case of (13), the r.h.s. of the second equation above is obviously well-defined since $Y_{\epsilon} \in C_{c}^{\infty}\left([0, T] \times \mathbf{R}^{3}\right)$ for each $T>0$.

Comparing the original dynamics (13) with its regularized analogue (27), one notices that, in (13) the force acting on the $i$-th particle is the electric or electromagnetic force created by the $N-1$ other particles. Self-interaction - i.e. the action of the electromagnetic field created by the $i$-th particle on itself - is therefore neglected in (13), unlike in the regularized model (27). As a matter of fact, there are serious conceptual difficulties with the notion of self-force in classical electrodynamics, which are beyond the scope of the present paper. The interested reader is referred to the discussion on pp. $675-676$ of [8] see also chapter 16 of [11] and especially [17] for more on this subject. That the self-interaction force is present in the regularized model (27) is only for mathematical convenience (see below).

In fact, if the regularizing parameter is kept fixed, neglecting the selfinteraction force in (27) will only produce an error of order $O(1 / N)$ over finite time intervals. 
Indeed, consider instead of (27) the following regularized variant of (13):

$$
\left\{\begin{array}{l}
\dot{\hat{x}}_{i}(t)=v\left(\hat{\xi}_{i}(t)\right) \\
\dot{\hat{\xi}}_{i}(t)=-\frac{1}{N-1} \sum_{\substack{j=1 \\
j \neq i}}^{N} \nabla_{x} \partial_{t} Y_{\epsilon}(t, \cdot) \star_{x} G\left(\hat{x}_{i}(0)-\hat{x}_{j}(0)\right) \\
-\frac{1}{N-1} \sum_{\substack{j=1 \\
j \neq i}}^{N} \int_{0}^{t}\left(\nabla_{x}+v\left(\hat{\xi}_{j}(s)\right) \partial_{t}\right) Y_{\epsilon}\left(t-s, \hat{x}_{i}(t)-\hat{x}_{j}(s)\right) d s \\
-\frac{1}{N-1} \sum_{\substack{j=1 \\
j \neq i}}^{N} \int_{0}^{t} v\left(\hat{\xi}_{i}(t)\right) \wedge\left(v\left(\hat{\xi}_{j}(s)\right) \wedge \nabla_{x} Y_{\epsilon}\left(t-s, \hat{x}_{i}(t)-\hat{x}_{j}(s)\right)\right) d s . \\
i=1, \ldots, N .
\end{array}\right.
$$

That the regularized dynamics defined by (27) and (28) are equivalent on finite time intervals as $N \rightarrow+\infty$ is summarized in the following statement.

Proposition 3.2. Let $\epsilon, T>0$ and $N \geq 2$. If $\left(x_{i}, \xi_{i}\right)_{1 \leq i \leq N}$ and $\left(\hat{x}_{i}, \hat{\xi}_{i}\right)_{1 \leq i \leq N}$ are solutions of (27) and of (28) respectively on $[0, T]$, and if

$$
x_{i}(0)=\hat{x}_{i}(0), \quad \xi_{i}(0)=\hat{\xi}_{i}(0) \quad \text { for } i=1, \ldots, N,
$$

then one has the error estimate

$$
\left|\hat{x}_{i}(t)-x_{i}(t)\right|+\left|\hat{\xi}_{i}(t)-\xi_{i}(t)\right|=O(1 / N)
$$

for all $i=1, \ldots, N$ and all $t \in[0, T]$. 
Proof. Observe that, for each $N \geq 2$ and

$$
\begin{aligned}
& \mid \frac{1}{N-1} \sum_{\substack{j=1 \\
j \neq i}}^{N} \int_{0}^{t}\left(\nabla_{x}+v\left(\hat{\xi}_{j}(s)\right) \partial_{t}\right) Y_{\epsilon}\left(t-s, \hat{x}_{i}(t)-\hat{x}_{j}(s)\right) d s \\
& \quad-\frac{1}{N} \sum_{j=1}^{N} \int_{0}^{t}\left(\nabla_{x}+v\left(\xi_{j}(s)\right) \partial_{t}\right) Y_{\epsilon}\left(t-s, x_{i}(t)-x_{j}(s)\right) d s \mid \\
& \leq \frac{1}{N(N-1)} \sum_{\substack{j=1 \\
j \neq i}}^{N} \int_{0}^{t}\left|\left(\nabla_{x}+v\left(\hat{\xi}_{j}(s)\right) \partial_{t}\right) Y_{\epsilon}\left(t-s, \hat{x}_{i}(t)-\hat{x}_{j}(s)\right)\right| d s \\
& +\frac{1}{N} \sum_{\substack{j=1 \\
j \neq i}}^{N} \int_{0}^{t} \mid\left(\nabla_{x}+v\left(\hat{\xi}_{j}(s)\right) \partial_{t}\right) Y_{\epsilon}\left(t-s, \hat{x}_{i}(t)-\hat{x}_{j}(s)\right) \\
& +\frac{1}{N} \int_{0}^{t}\left|\left(\nabla_{x}+v\left(\xi_{i}(s)\right) \partial_{t}\right) Y_{\epsilon}\left(t-s, x_{i}(t)-x_{i}(s)\right)\right| d s \\
& \leq \frac{2 t}{N-1}\left\|\nabla_{t, x} Y_{\epsilon}\right\|_{L^{\infty}\left([0, T] \times \mathbf{R}^{3}\right)} \\
& +\frac{1}{N} \sum_{\substack{j=1 \\
j \neq i}}^{N}\left\|\nabla_{t, x}^{2} Y_{\epsilon}\right\|_{L^{\infty}\left([0, T] \times \mathbf{R}^{3}\right)} \int_{0}^{t}\left|\hat{x}_{j}(s)-x_{j}(s)\right| d s \\
& +\frac{1}{N} \sum_{\substack{j=1 \\
j \neq i}}^{N}\left\|\partial_{t} Y_{\epsilon}\right\|_{L^{\infty}\left([0, T] \times \mathbf{R}^{3}\right)}\left\|\nabla_{\xi} v\right\|_{L^{\infty}\left(\mathbf{R}^{3}\right)} \int_{0}^{t}\left|\hat{\xi}_{j}(s)-\xi_{j}(s)\right| d s \\
& +2 T\left\|\nabla_{t, x}^{2} Y_{\epsilon}\right\|_{L^{\infty}\left([0, T] \times \mathbf{R}^{3}\right)}\left|\hat{x}_{i}(t)-x_{i}(t)\right| . \\
&
\end{aligned}
$$

The difference between the right-hand sides of the equations for $\dot{\xi}_{i}$ in (27) and (28) involves two more terms analogous to this one.

Thus, denoting

$$
\mathcal{E}_{i}(t):=\left|\hat{x}_{i}(t)-x_{i}(t)\right|+\left|\hat{\xi}_{i}(t)-\xi_{i}(t)\right|
$$


we see that, for each $i=1, \ldots, N$ and all $t \in[0, T]$, one has

$$
\begin{array}{r}
\mathcal{E}_{i}(t) \leq C_{\epsilon}\left(\int_{0}^{t} \mathcal{E}_{i}(\tau) d \tau+\frac{1}{N} \sum_{j=1}^{N} \int_{0}^{t} \int_{0}^{\tau} \mathcal{E}_{i}(s) d s d \tau+\frac{4 t+2}{N}\right) \\
=C_{\epsilon}\left(\int_{0}^{t} \mathcal{E}_{i}(\tau) d \tau+\frac{1}{N} \sum_{j=1}^{N} \int_{0}^{t}(t-s) \mathcal{E}_{i}(s) d s+\frac{4 t+2}{N}\right) \\
\leq C_{\epsilon}\left(\int_{0}^{t} \mathcal{E}_{i}(\tau) d \tau+\frac{T}{N} \sum_{j=1}^{N} \int_{0}^{t} \mathcal{E}_{i}(s) d s+\frac{4 T+2}{N}\right),
\end{array}
$$

since $\mathcal{E}_{i}(0)=0$, where $C_{\epsilon}$ is a constant depending on

$$
\begin{array}{r}
\left\|\nabla_{t, x} Y_{\epsilon}\right\|_{L^{\infty}\left([0, T] \times \mathbf{R}^{3}\right)},\left\|\nabla_{t, x}^{2} Y_{\epsilon}\right\|_{L^{\infty}\left([0, T] \times \mathbf{R}^{3}\right)},\left\|G \star_{x} \nabla_{x} Y_{\epsilon}\right\|_{L^{\infty}\left([0, T] \times \mathbf{R}^{3}\right)} \\
\text { and }\left\|\nabla_{\xi} v\right\|_{L^{\infty}\left(\mathbf{R}^{3}\right)} .
\end{array}
$$

Therefore, summing for $i=1, \ldots, N$ both sides of the inequality above shows that

$$
\mathcal{E}(t):=\frac{1}{N} \sum_{i=1}^{N} \mathcal{E}_{i}(t) \leq C_{\epsilon}\left((1+T) \int_{0}^{t} \mathcal{E}(s) d s+\frac{4 T+2}{N}\right)
$$

and we conclude from Gronwall's inequality that

$$
\mathcal{E}(t) \leq C_{\epsilon} \frac{4 T+2}{N} e^{C_{\epsilon}(1+T) t}, \quad 0 \leq t \leq T .
$$

Substituting this in the inequality satisfied by $\mathcal{E}_{i}$, we find that

$$
\begin{array}{r}
\mathcal{E}_{i}(t) \leq C_{\epsilon}\left(\int_{0}^{t} \mathcal{E}_{i}(\tau) d \tau+T \int_{0}^{t} \mathcal{E}(s) d s+\frac{4 T+2}{N}\right) \\
\leq C_{\epsilon} \int_{0}^{t} \mathcal{E}_{i}(\tau) d \tau+\frac{4 T}{N} e^{C_{\epsilon}(1+T) t}+\frac{2}{N}
\end{array}
$$

so that, applying again Gronwall's inequality leads to

$$
\mathcal{E}_{i}(t) \leq \frac{2}{N}\left(1+2 T e^{C_{\epsilon}(1+T) T}\right) e^{C_{\epsilon} t}, \quad 0 \leq t \leq T, i=1, \ldots, N .
$$

\section{Dobrushin's estimate}

Let $k$ be a $\mathbf{R}^{n}$-valued Radon measure on $\mathbf{R}^{1+d} \times \mathbf{R}^{1+d}$ of the form

$$
k(t, z, \tau, \zeta)=m(t, z, \zeta) \delta_{\tau=0}+r(t, z, \tau, \zeta), \quad z, \zeta \in \mathbf{R}^{d}, s, t \geq 0 .
$$


We assume that $r \in C_{b}\left(\mathbf{R}^{1+d} \times \mathbf{R}^{1+d} ; \mathbf{R}^{n}\right)$ and $m \in C_{b}\left(\mathbf{R}^{1+d} \times \mathbf{R}^{d} ; \mathbf{R}^{n}\right)$ satisfy the properties

$$
\begin{array}{cl}
r(t, z, \tau, \zeta)=0 & \text { whenever } \tau \notin[0, t] \text { and } z, \zeta \in \mathbf{R}^{d}, \\
m(t, z, \zeta)=0 & \text { whenever } t<0 \text { and } z, \zeta \in \mathbf{R}^{d},
\end{array}
$$

together with the Lipschitz condition

$$
\begin{aligned}
& \left|r(t, z, \tau, \zeta)-r\left(t, z^{\prime}, \tau, \zeta^{\prime}\right)\right|+\left|m\left(t, z, \zeta_{0}\right)-r\left(t, z^{\prime}, \zeta_{0}^{\prime}\right)\right| \\
& \quad \leq \operatorname{Lip}_{T}(k)\left(1 \wedge\left|z-z^{\prime}\right|+1 \wedge\left|\zeta-\zeta^{\prime}\right|+1 \wedge\left|\zeta_{0}-\zeta_{0}^{\prime}\right|\right)
\end{aligned}
$$

for all $t, t^{\prime}, \tau, \tau^{\prime} \in[0, T]$ and all $z, z^{\prime}, \zeta, \zeta^{\prime}, \zeta_{0}, \zeta_{0}^{\prime} \in \mathbf{R}^{d}$, where the notation $a \wedge b$ designates $\min (a, b)$ for all $a, b \in \mathbf{R}$.

We consider the integral operator with kernel $k$, i.e.

$$
K \mu(t, z)=\int_{\mathbf{R}^{1+d}} r(t, z, \tau, \zeta) \mu(\tau, d \zeta) d \tau+\int_{\mathbf{R}^{d}} m(t, z, \zeta) \mu(0, d \zeta)
$$

for each $\mu \in C\left(\mathbf{R}_{+} ; w-\mathcal{P}\left(\mathbf{R}^{d}\right)\right)$, where $w-\mathcal{P}\left(\mathbf{R}^{d}\right)$ designates the set of Borel probability measures on $\mathbf{R}^{d}$ equipped with its weak topology.

For any given Borel probability measure $\rho^{i n}$ on $\mathbf{R}^{d}$, consider $Z \equiv$ $Z\left(t, z ; \rho^{i n}\right)$, the solution of the mean-field integro-differential equation

$$
\left\{\begin{array}{l}
\dot{Z}=K \rho(t, Z) \\
\rho(t, \cdot)=Z\left(t, \cdot ; \rho^{i n}\right) \# \rho^{i n} \\
Z\left(0, z ; \rho^{i n}\right)=z
\end{array}\right.
$$

(If $\Phi: \mathbf{R}^{d} \mapsto \mathbf{R}^{d}$ is a Borel transformation and $\rho$ a Borel probability measure on $\mathbf{R}^{d}$, the notation $\Phi \# \rho$ designates the image measure of $\rho$ under $\Phi$, defined by the formula $\Phi \# \rho(A)=\rho\left(\Phi^{-1}(A)\right)$ for each Borel set $A \subset \mathbf{R}^{d}$.)

Proposition 4.1. For each Borel probability measure $\rho^{\text {in }}$ on $\mathbf{R}^{d}$, the mean-field integro-differential equation (32) has a unique global solution $Z \in C\left(\mathbf{R}_{+} \times \mathbf{R}^{d} ; \mathbf{R}^{d}\right)$ such that $t \mapsto Z(t, z)$ is continuously differentiable on $\mathbf{R}_{+}$for each $z \in \mathbf{R}^{d}$.

Proof. To any bounded map $Z$ belonging to $C\left(\mathbf{R}_{+} \times \mathbf{R}^{d} ; \mathbf{R}^{d}\right)$, we associate the map $\tilde{Z}$ in $C\left(\mathbf{R}_{+} \times \mathbf{R}^{d} ; \mathbf{R}^{d}\right)$ defined by

$$
\begin{aligned}
\tilde{Z}(t, z)=z+\int_{0}^{t} K\left(Z(\cdot, \cdot) \# \rho^{i n}\right)(\tau, Z(\tau, z)) d \tau \\
=z+\int_{0}^{t} \int_{0}^{s} \int_{\mathbf{R}^{d}} r(s, Z(s, z), \tau, Z(\tau, \zeta)) \rho^{i n}(d \zeta) d \tau d s \\
\quad+\int_{0}^{t} \int_{\mathbf{R}^{d}} m(s, Z(s, z), \zeta) \rho^{i n}(d \zeta) d s .
\end{aligned}
$$


Let $Z_{1}, Z_{2} \in C\left(\mathbf{R}_{+} \times \mathbf{R}^{d} ; \mathbf{R}^{d}\right)$ and denote $\tilde{Z}_{1}, \tilde{Z}_{2}$ the maps so defined in terms of $Z_{1}, Z_{2}$. Then, by the Lipschitz condition (31)

$$
\begin{aligned}
\mid \tilde{Z}_{1}(t, z)- & \tilde{Z}_{2}(t, z) \mid \\
& \leq \operatorname{Lip}_{T}(k) \int_{0}^{t} \int_{0}^{s} \int_{\mathbf{R}^{d}}\left|Z_{1}(s, z)-Z_{2}(s, z)\right| \rho^{i n}(d \zeta) d \tau d s \\
& +\operatorname{Lip}_{T}(k) \int_{0}^{t} \int_{0}^{s} \int_{\mathbf{R}^{d}}\left|Z_{1}(\tau, \zeta)-Z_{2}(\tau, \zeta)\right| \rho^{i n}(d \zeta) d \tau d s \\
& +\operatorname{Lip}_{T}(k) \int_{0}^{t} \int_{\mathbf{R}^{d}}\left|Z_{1}(s, z)-Z_{2}(s, z)\right| \rho^{i n}(d \zeta) d s,
\end{aligned}
$$

for all $t \in[0, T]$. Denote

$$
\left\{\begin{array}{l}
\mathcal{E}(t):=\sup _{z \in \mathbf{R}^{N}}\left|Z_{1}(t, z)-Z_{2}(t, z)\right| \\
\tilde{\mathcal{E}}(t):=\sup _{z \in \mathbf{R}^{N}}\left|\tilde{Z}_{1}(t, z)-\tilde{Z}_{2}(t, z)\right|
\end{array}\right.
$$

for all $t \geq 0$. Then, for all $t \in[0, T]$, one has

$$
\begin{array}{r}
\tilde{\mathcal{E}}(t) \leq \operatorname{Lip}_{T}(k)\left(\int_{0}^{t}(1+s) \mathcal{E}(s) d s+\int_{0}^{t} \int_{0}^{s} \mathcal{E}(\tau) d \tau d s\right) \\
=\operatorname{Lip}_{T}(k)\left(\int_{0}^{t}(1+s) \mathcal{E}(s) d s+\int_{0}^{t}(t-\tau) \mathcal{E}(\tau) d \tau\right) \\
=\operatorname{Lip}_{T}(k)(1+t) \int_{0}^{t} \mathcal{E}(s) d s .
\end{array}
$$

With this estimate, we construct the solution by the usual Picard iteration procedure. Define recursively a sequence of maps $\left(Z^{n}\right)_{n \geq 0}$ by

$$
\left\{\begin{array}{l}
Z^{n+1}(t, z):=z+\int_{0}^{t} K\left(Z^{n}(\cdot, \cdot) \# \rho^{i n}\right)\left(\tau, Z^{n}(\tau, z)\right) d \tau, \quad t \geq 0 \\
Z^{0}(t, z):=z
\end{array}\right.
$$

Applying (33) iteratively with the notation

$$
\mathcal{E}^{n}(t)=\sup _{z \in \mathbf{R}^{d}}\left|Z^{n+1}(t, z)-Z^{n}(t, z)\right|
$$


shows that, for all $t \in[0, T]$, one has

$$
\begin{aligned}
\mathcal{E}^{n}(t) & \leq \operatorname{Lip}_{T}(k)(1+t) \int_{0}^{t} \mathcal{E}^{n-1}\left(t_{1}\right) d t_{1} \\
& \leq \operatorname{Lip}_{T}(k)^{2}(1+t) \int_{0}^{t}\left(1+t_{1}\right) \int_{0}^{t_{1}} \mathcal{E}^{n-2}\left(t_{2}\right) d t_{2} d t_{1} \\
& \leq \operatorname{Lip}_{T}(k)^{2}(1+t)^{2} \int_{0}^{t} \int_{0}^{t_{1}} \mathcal{E}^{n-2}\left(t_{2}\right) d t_{2} d t_{1} \\
& \ldots \\
& \leq \operatorname{Lip}_{T}(k)^{n}(1+t)^{n} \frac{t^{n}}{n !} \sup _{0 \leq t_{n} \leq t} \mathcal{E}^{0}\left(t_{n}\right)
\end{aligned}
$$

since

$$
\int \mathbf{1}_{0 \leq t_{n} \leq t_{n-1} \leq \ldots \leq t_{1} \leq t} d t_{n} d t_{n-1} \ldots d t_{1}=\frac{t^{n}}{n !}
$$

Since

$$
\sum_{n \geq 0} \frac{\operatorname{Lip}_{T}(k)^{n}(1+t)^{n} t^{n}}{n !}<+\infty
$$

for each $t>0$, we conclude that the sequence $Z^{n}$ converges uniformly on $[0, T] \times \mathbf{R}^{d}$ for each $T>0$ towards a solution of the integral equation

$$
Z(t, z)=z+\int_{0}^{t} K\left(Z(\cdot, \cdot) \# \rho^{i n}\right)(\tau, Z(\tau, z)) d \tau
$$

defined for each $t \geq 0$ and such that $Z \in C\left(\mathbf{R}_{+} \times \mathbf{R}^{d} ; \mathbf{R}^{d}\right)$.

If $Z_{1}$ and $Z_{2}$ are two solutions of that integral equation, denoting as above

$$
\mathcal{E}(t)=\sup _{z \in \mathbf{R}^{d}}\left|Z_{1}(t, z)-Z_{2}(t, z)\right|,
$$

we conclude from (33) that

$$
\mathcal{E}(t) \leq \operatorname{Lip}_{T}(k)(1+t) \int_{0}^{t} \mathcal{E}(s) d s
$$

and, following the argument in (34), that

$$
\sup _{0 \leq t \leq T} \mathcal{E}(t) \leq \operatorname{Lip}_{T}(k)^{n}(1+T)^{n} \frac{T^{n}}{n !} \sup _{0 \leq t \leq T} \mathcal{E}(t) .
$$

Letting $n \rightarrow+\infty$ while keeping $T>0$ fixed shows that $\mathcal{E}(t)=0$ for each $t \in[0, T]$; and since $T>0$ is arbitrary, $Z_{1}=Z_{2}$ on $\mathbf{R}_{+} \times \mathbf{R}^{d}$. This establishes the uniqueness of the solution $Z$ of the integral equation (35). 
Finally, since the integral kernels $r$ and $m$ are bounded continuous functions of all their arguments while $\rho^{i n}$ is a probability measure on $\mathbf{R}^{d}$, applying the dominated convergence theorem shows that $t \mapsto K\left(Z(\cdot, \cdot) \# \rho^{i n}\right)(t, z)$ is continuous for all $z \in \mathbf{R}^{d}$, so that $t \mapsto Z(t, z)$ is continuously differentiable in $t$ for all $z \in \mathbf{R}^{d}$ and satisfies the differential form (32) of the integral equation (35).

Once the existence and uniqueness of the solution of the mean-field equation (32) is known, the next natural question is whether its dependence in the initial probability measure $\rho^{i n}$ is continuous. The right topology in which to measure the proximity of two initial probability densities $\rho_{1}^{i n}, \rho_{2}^{i n}$ is obviously that of the weak convergence of probability measures on $\mathbf{R}^{d}$. An extremely convenient way to obtain quantitative information in that topology is to use the Monge-KantorovichRubinstein distance, whose definition we recall below.

Definition 4.2. Let $\mathcal{P}\left(\mathbf{R}^{d}\right)$ be the set of Borel probability measures on $\mathbf{R}^{d}$. The Monge-Kantorovich-Rubinstein distance on $\mathcal{P}\left(\mathbf{R}^{d}\right)$ is defined by the formula

$$
\operatorname{dist}_{M K R}(\mu, \nu)=\inf _{\pi \in \Pi(\mu, \nu)} \iint_{\mathbf{R}^{d} \times \mathbf{R}^{d}} 1 \wedge|x-y| \pi(d x d y)
$$

where $\Pi(\mu, \nu)$ is the set of Borel probability measures on $\mathbf{R}^{d} \times \mathbf{R}^{d}$ whose marginals are $\mu$ and $\nu$, i.e.

$$
\iint_{\mathbf{R}^{d} \times \mathbf{R}^{d}} \phi(x) \psi(y) \pi(d x d y)=\int_{\mathbf{R}^{d}} \phi(x) \mu(d x) \int_{\mathbf{R}^{d}} \psi(y) \nu(d y)
$$

for all bounded continuous functions $\phi, \psi$ defined on $\mathbf{R}^{d}$.

This distance is also called the Wasserstein distance - although it was considered more than ten years earlier by Kantorovich and Rubinstein, and appears in the work of Monge.

Our (slight) generalization of the Dobrushin estimate presented in [7] is stated in the following proposition.

Proposition 4.3. Let $\rho_{1}^{\text {in }}$ and $\rho_{2}^{\text {in }}$ be two Borel probability measures on $\mathbf{R}^{d}$, and let $Z_{1}, Z_{2}$ be the corresponding solutions of (32), denoted

$$
Z_{j}(t, z):=Z\left(t, z ; \rho_{j}^{\text {in }}\right) \text { for } j=1,2, \quad t \geq 0, \text { and } z \in \mathbf{R}^{d} \text {, }
$$

whose existence and uniqueness is established by Proposition 4.1, and

$$
\rho_{j}(t, \cdot):=Z_{j}(t, \cdot) \# \rho_{j}^{i n}, \quad \text { for } j=1,2 .
$$

Then, for each $t \geq 0$,

$$
\operatorname{dist}_{M K R}\left(\rho_{1}(t, \cdot), \rho_{2}(t, \cdot)\right) \leq\left(1+t \operatorname{Lip}_{t}(k)\right) e^{t^{2} \operatorname{Lip}_{t}(k)} \operatorname{dist}_{M K R}\left(\rho_{1}^{i n}, \rho_{2}^{i n}\right) .
$$


The main difference between this result and Proposition 4 in [7] is that the proposition above applies to an integro-differential equation of the form (32) satisfying the causality assumption (30), whereas the differential system considered in Dobrushin's original contribution [7] did not involve memory effects. This is only natural since Dobrushin was interested in the Vlasov-Poisson equation, a non-relativistic model in which the force field is created by the instantaneous distribution of charges.

There are other minor differences: for instance, unlike Dobrushin's the estimate obtained here is local in time and uses the Gronwall inequality; besides, the integral operator $K$ in (32) considered in the proposition above is not necessarily a convolution operator as in [7]. But the argument is mostly the same as in [7], and we give it below only for the sake of being complete.

Proof. One has

$$
\begin{aligned}
K \rho_{1}( & \left.t, Z_{1}\left(t, z_{1}\right)\right)-K \rho_{2}\left(t, Z_{2}\left(t, z_{2}\right)\right) \\
= & \int_{0}^{t} \int_{\mathbf{R}^{d}} r\left(t, Z_{1}\left(t, z_{1}\right), \tau, \zeta_{1}\right) \rho_{1}\left(\tau, d \zeta_{1}\right) d \tau \\
& +\int_{\mathbf{R}^{d}} m\left(t, Z_{1}\left(t, z_{1}\right), \zeta_{1}\right) \rho_{1}^{i n}\left(d \zeta_{1}\right) \\
& -\int_{0}^{t} \int_{\mathbf{R}^{d}} r\left(t, Z_{2}\left(t, z_{2}\right), \tau, \zeta_{2}\right) \rho_{2}\left(\tau, d \zeta_{2}\right) d \tau \\
& -\int_{\mathbf{R}^{d}} m\left(t, Z_{2}\left(t, z_{2}\right), \zeta_{2}\right) \rho_{2}^{i n}\left(d \zeta_{2}\right) \\
= & \int_{0}^{t} \int_{\mathbf{R}^{d}} r\left(t, Z_{1}\left(t, z_{1}\right), \tau, Z_{1}\left(\tau, \zeta_{1}\right)\right) \rho_{1}^{i n}\left(d \zeta_{1}\right) d \tau \\
+ & \int_{\mathbf{R}^{d}} m\left(t, Z_{1}\left(t, z_{1}\right), \zeta_{1}\right) \rho_{1}^{i n}\left(d \zeta_{1}\right) \\
- & \int_{0}^{t} \int_{\mathbf{R}^{d}} r\left(t, Z_{2}\left(t, z_{2}\right), \tau, Z_{2}\left(\tau, \zeta_{2}\right)\right) \rho_{2}^{i n}\left(d \zeta_{2}\right) d \tau \\
- & \int_{\mathbf{R}^{d}} m\left(t, Z_{2}\left(t, z_{2}\right), \zeta_{2}\right) \rho_{2}^{i n}\left(d \zeta_{2}\right)
\end{aligned}
$$

for each $t \geq 0$ and $z_{1}, z_{2} \in \mathbf{R}^{d}$. In the second equality above, we have used the identity

$$
\int_{\mathbf{R}^{d}} \phi\left(Z_{j}\left(\tau, \zeta_{j}\right)\right) \rho_{j}^{i n}\left(d \zeta_{j}\right)=\int_{\mathbf{R}^{d}} \phi\left(\zeta_{j}\right) \rho_{j}\left(\tau, d \zeta_{j}\right)
$$


for all bounded $\phi$, Borel measurable on $\mathbf{R}^{d}$, since $\rho_{j}(\tau, \cdot)=Z_{j}(\tau, \cdot) \# \rho_{j}^{i n}$ - see the definition of the image of a probability measure under a Borel map following (32), that is equivalent to (36) with $\phi=\mathbf{1}_{A}$.

Pick $\pi^{i n}$ to be any probability measure on $\mathbf{R}^{d} \times \mathbf{R}^{d}$ with $\rho_{1}^{\text {in }}$ and $\rho_{2}^{\text {in }}$ as marginals: one has, for each $t \geq 0$ and $z_{1}, z_{2} \in \mathbf{R}^{d}$

$$
\begin{aligned}
& K \rho_{1}\left(t, Z_{1}\left(t, z_{1}\right)\right)-K \rho_{2}\left(t, Z_{2}\left(t, z_{2}\right)\right) \\
& =\int_{0}^{t} \iint_{\mathbf{R}^{d} \times \mathbf{R}^{d}}\left(r\left(t, Z_{1}\left(t, z_{1}\right), \tau, Z_{1}\left(\tau, \zeta_{1}\right)\right)\right. \\
& \left.\quad-r\left(t, Z_{2}\left(t, z_{2}\right), \tau, Z_{2}\left(\tau, \zeta_{2}\right)\right)\right) \pi^{i n}\left(d \zeta_{1}, d \zeta_{2}\right) \\
& \quad+\iint_{\mathbf{R}^{d} \times \mathbf{R}^{d}}\left(m\left(t, Z_{1}\left(t, z_{1}\right), \zeta_{1}\right)-m\left(t, Z_{2}\left(t, z_{2}\right), \zeta_{2}\right)\right) \pi^{i n}\left(d \zeta_{1}, d \zeta_{2}\right) .
\end{aligned}
$$

Hence, as a consequence of (31), for each $t \in[0, T]$ and $z_{1}, z_{2} \in \mathbf{R}^{d}$

$$
\begin{aligned}
& \left|K \rho_{1}\left(t, Z_{1}\left(t, z_{1}\right)\right)-K \rho_{2}\left(t, Z_{2}\left(t, z_{2}\right)\right)\right| \\
& \quad \leq \operatorname{Lip}_{T}(k)\left(t 1 \wedge\left|Z_{1}\left(t, z_{1}\right)-Z_{2}\left(t, z_{2}\right)\right|+\int_{0}^{t} \mathcal{D}(\tau) d \tau+\mathcal{D}(0)\right)
\end{aligned}
$$

with the notation

$$
\mathcal{D}(\tau):=\iint_{\mathbf{R}^{d} \times \mathbf{R}^{d}} 1 \wedge\left|Z_{1}\left(\tau, \zeta_{1}\right)-Z_{2}\left(\tau, \zeta_{2}\right)\right| \pi^{i n}\left(d \zeta_{1}, d \zeta_{2}\right)
$$

Therefore, for each $t \in[0, T]$ and each $z_{1}, z_{2} \in \mathbf{R}^{d}$, one has

$$
\begin{aligned}
& \left|Z_{1}\left(t, z_{1}\right)-Z_{2}\left(t, z_{2}\right)\right| \\
& \leq\left|z_{1}-z_{2}\right|+\int_{0}^{t}\left|K \rho_{1}\left(s, Z_{1}\left(s, z_{1}\right)\right)-K \rho_{2}\left(s, Z_{2}\left(s, z_{2}\right)\right)\right| d s \\
& \leq\left|z_{1}-z_{2}\right|+\operatorname{Lip}_{T}(k) t \mathcal{D}(0) \\
& +\operatorname{Lip}_{T}(k)\left(\int_{0}^{t} s 1 \wedge\left|Z_{1}\left(s, z_{1}\right)-Z_{2}\left(s, z_{2}\right)\right| d s+\int_{0}^{t} \int_{0}^{s} \mathcal{D}(\tau) d \tau d s\right) .
\end{aligned}
$$


Integrating with the measure $\pi^{i n}\left(d z_{1} d z_{2}\right)$ both sides of the resulting inequality for $1 \wedge\left|Z_{1}\left(t, z_{1}\right)-Z_{2}\left(t, z_{2}\right)\right|$, we arrive at

$$
\begin{aligned}
\mathcal{D}(t) & \leq\left(1+t \operatorname{Lip}_{T}(k)\right) \mathcal{D}(0) \\
& +\operatorname{Lip}_{T}(k)\left(\int_{0}^{t} s \mathcal{D}(s) d s+\int_{0}^{t} \int_{0}^{s} \mathcal{D}(\tau) d \tau d s\right) \\
& =\left(1+t \operatorname{Lip}_{T}(k)\right) \mathcal{D}(0) \\
& +\operatorname{Lip}_{T}(k)\left(\int_{0}^{t} s \mathcal{D}(s) d s+\int_{0}^{t}(t-s) \mathcal{D}(s) d s\right) \\
& =\left(1+t \operatorname{Lip}_{T}(k)\right) \mathcal{D}(0)+\operatorname{Lip}_{T}(k) t \int_{0}^{t} \mathcal{D}(s) d s .
\end{aligned}
$$

This implies the estimate

$$
\mathcal{D}(t) \leq \mathcal{D}(0) \Psi(t), \quad t \geq 0,
$$

where

$$
\Psi(t)=\left(1+t \operatorname{Lip}_{t}(k)\right) e^{t^{2} \operatorname{Lip}_{t}(k)}, \quad t \geq 0
$$

Now

$$
\mathcal{D}(t)=\iint_{\mathbf{R}^{d} \times \mathbf{R}^{d}} 1 \wedge\left|\zeta_{1}-\zeta_{2}\right| \pi\left(t ; d \zeta_{1} d \zeta_{2}\right)
$$

where

$$
\pi(t, \cdot):=\left(Z_{1}(t, \cdot), Z_{2}(t, \cdot)\right) \# \pi^{i n}
$$

is the image under the map $\left(z_{1}, z_{2}\right) \mapsto\left(Z_{1}\left(t, z_{1}\right), Z_{2}\left(t, z_{2}\right)\right)$ of the measure $\pi^{i n}$. Obviously

$$
\pi^{i n} \in \Pi\left(\rho_{1}^{i n}, \rho_{2}^{i n}\right) \Rightarrow \pi(t, \cdot) \in \Pi\left(\rho_{1}(t, \cdot), \rho_{2}(t, \cdot)\right)
$$

for each $t \geq 0$, so that

$$
\operatorname{dist}_{M K R}\left(\rho_{1}(t, \cdot), \rho_{2}(t, \cdot)\right) \leq \Psi(t) \mathcal{D}(0) .
$$

Minimizing the right-hand side of this inequality as $\pi^{i n}$ runs through $\Pi\left(\rho_{1}^{i n}, \rho_{2}^{i n}\right)$ leads to the desired estimate.

\section{Application to the Regularized Vlasov-Maxwell DYNAMICS}

Let $\epsilon>0$ be a fixed regularization parameter, and consider the regularized Vlasov-Maxwell system (17). Henceforth we assume that $f^{\text {in }}$ is a probability density on $\mathbf{R}^{3} \times \mathbf{R}^{3}$. Applying the method of characteristics to the transport equation governing $f_{\epsilon}$ shows that

$$
f_{\epsilon}\left(t, X_{\epsilon}\left(t, x, v ; f^{i n}\right), \Xi_{\epsilon}\left(t, x, v ; f^{i n}\right)\right)=f^{i n}(x, v)
$$


F. GOLSE

where $t \mapsto\left(X_{\epsilon}, \Xi_{\epsilon}\right)\left(t, \cdot, \cdot ; f^{i n}\right)$ is the solution of

$$
\left\{\begin{aligned}
\dot{X}_{\epsilon} & =v\left(\Xi_{\epsilon}\right) \\
\dot{\Xi}_{\epsilon} & =E_{\epsilon}\left(t, X_{\epsilon}\right)+v\left(\Xi_{\epsilon}\right) \wedge B_{\epsilon}\left(t, X_{\epsilon}\right) \\
& =F_{\epsilon}\left[f_{\epsilon}(t, \cdot, \cdot)\right]\left(X_{\epsilon}, \Xi_{\epsilon}\right),
\end{aligned}\right.
$$

with the notation

$$
\begin{aligned}
F_{\epsilon}[f]:= & -\int_{\mathbf{R}^{3}} \partial_{t} \nabla_{x} Y_{\epsilon}(t, \cdot) \star_{x} G \star_{x} f(0, \cdot, \eta) d \eta \\
& -\int_{\mathbf{R}^{3}}\left(\nabla_{x}+v(\eta) \partial_{t}\right) Y_{\epsilon} \star_{t, x} f(\cdot, \cdot, \eta) d \eta \\
& -\int_{\mathbf{R}^{3}} v(\xi) \wedge\left(v(\eta) \wedge \nabla_{x} Y_{\epsilon} \star_{t, x} f(\cdot, \cdot, \eta)\right) d \eta .
\end{aligned}
$$

Since $\operatorname{div}_{x, \xi}\left(v(\xi), E_{\epsilon}(t, x)+v(\xi) \wedge B_{\epsilon}(t, x)\right)=0$, the flow $\left(X_{\epsilon}, \Xi_{\epsilon}\right)$ leaves the Lebesgue measure $d x d \xi$ invariant so that (37) can be recast as

$$
f_{\epsilon}(t, \cdot, \cdot) d x d \xi=\left(X_{\epsilon}, \Xi_{\epsilon}\right)\left(t, \cdot, \cdot ; f^{i n}\right) \# f^{i n} d x d \xi .
$$

According to (39), the vector field $\left(v(\xi), E_{\epsilon}(t, x)+v(\xi) \wedge B_{\epsilon}(t, x)\right)$ can be expressed by an integral operator with kernel of the form (29) satisfying the causality and Lipschitz conditions (30) and (31):

$$
\begin{aligned}
\left(v(\xi), E_{\epsilon}(t, x)\right. & \left.+v(\xi) \wedge B_{\epsilon}(t, x)\right)=K_{\epsilon}\left(\left(X_{\epsilon}, \Xi_{\epsilon}\right)\left(t, \cdot, \cdot ; f^{i n}\right) \# f^{i n}\right)(t, x, \xi) \\
& =\int_{0}^{t} \iint_{\mathbf{R}^{3} \times \mathbf{R}^{3}} r_{\epsilon}(t, x, \xi, \tau, y, \eta) f_{\epsilon}(\tau, y, \eta) d y d \eta d \tau \\
& +\iint_{\mathbf{R}^{3} \times \mathbf{R}^{3}} m_{\epsilon}(t, x, \xi, y, \eta) f^{i n}(y, \eta) d y d \eta .
\end{aligned}
$$

In this formula, $r_{\epsilon}=\left(r_{\epsilon}^{1}, r_{\epsilon}^{2}\right)$ and $m_{\epsilon}=\left(m_{\epsilon}^{1}, m_{\epsilon}^{2}\right)$, where $r_{\epsilon}^{1}, r_{\epsilon}^{2}, m_{\epsilon}^{1}$ and $m_{\epsilon}^{2}$ are given by

$$
\begin{aligned}
r_{\epsilon}^{1}(t, x, \xi, s, y, \eta) & =0 \\
m_{\epsilon}^{1}(t, x, \xi, y, \eta) & =\mathbf{1}_{t \geq 0} v(\xi), \\
r_{\epsilon}^{2}(t, x, \xi, s, y, \eta) & =-\left(\nabla_{x}+v(\eta) \partial_{t}\right) Y_{\epsilon}(t-s, x-y) \\
& -v(\xi) \wedge\left(v(\eta) \wedge \nabla_{x} Y_{\epsilon}(t-s, x-y)\right), \\
m_{\epsilon}^{2}(t, x, \xi, y, \eta) & =-\partial_{t} \nabla_{x} Y_{\epsilon} \star_{x} G(t, x-y) .
\end{aligned}
$$

Therefore the characteristic flow $Z_{\epsilon}:=\left(X_{\epsilon}, \Xi_{\epsilon}\right)$ of the regularized Vlasov-Maxwell system (17) satisfies a mean-field integro-differential system of the form (32), where the integral operator $K_{\epsilon}$ is defined by (41)-(42). That the integral kernel of $K_{\epsilon}$ satisfies (30) is obvious since 
$Y_{\epsilon}(t, x)=0$ for all $x \in \mathbf{R}^{3}$ if $t<0$. That it also satisfies the Lipschitz condition (31) is equally obvious in view of (26). Henceforth, we denote

$$
L(T, \epsilon)=\operatorname{Lip}_{T}\left(k_{\epsilon}\right)
$$

where $k_{\epsilon}$ is the integral kernel of the operator $K_{\epsilon}$ defined above.

Bringing together the results obtained in the previous sections, we finally obtain the regularized Vlasov-Maxwell system (21) — or equivalently (17) as the mean field limit of the $N$-particle system (27) as $N \rightarrow+\infty$.

Theorem 5.1. Let $\epsilon>0$ and $f^{\text {in }} \in L^{\infty}\left(\mathbf{R}^{3} \times \mathbf{R}^{3}\right)$ be such that

$$
f^{\text {in }} \geq 0 \text { a.e. on } \mathbf{R}^{3} \times \mathbf{R}^{3}, \quad \operatorname{supp}\left(f^{i n}\right) \text { is compact, }
$$

and

$$
\iint_{\mathbf{R}^{3} \times \mathbf{R}^{3}} f^{i n}(x, \xi) d x d \xi=1 .
$$

(In other words, $f^{\text {in }}$ is an essentially bounded probability distribution on $\mathbf{R}^{3} \times \mathbf{R}^{3}$.)

Let $\left(f_{\epsilon}, u_{\epsilon}\right)$ be the solution of (17) with initial data (18) - or, equivalently $\left(f_{\epsilon}, E_{\epsilon}, B_{\epsilon}\right)$ the solution of (21) with initial data (22).

On the other hand, let $\left(x_{i, N}^{i n}, \xi_{i, N}^{i n}\right)_{1 \leq i \leq N}$ be a sequence (indexed by $N)$ of $2 N$-tuples in $\mathbf{R}^{3}$ such that the initial empirical measure of the $N$-particle system

$$
f_{N}^{i n}:=\frac{1}{N} \sum_{i=1}^{N} \delta_{x_{i, N}^{i n}} \otimes \delta_{\xi_{i, N}^{i n}} \rightarrow f^{i n} d x d \xi \text { weakly }
$$

as $N \rightarrow+\infty$. Then

a) for each $N \geq 1$, the system (27) has a unique solution defined for all $t \geq 0$ and denoted $t \mapsto\left(x_{i, N}(t), \xi_{i, N}(t)\right)_{1 \leq i \leq N}$.

Denote

$$
f_{N, \epsilon}(t, \cdot, \cdot):=\frac{1}{N} \sum_{i=1}^{N} \delta_{x_{i, N}(t)} \otimes \delta_{\xi_{i, N}(t)}
$$

the $N$-particle empirical measure at time $t$ for each $t \geq 0$. Then b) for each $T>0$,

$$
f_{N, \epsilon}(t, \cdot, \cdot) \rightarrow f_{\epsilon}(t, x, \xi) d x d \xi \text { weakly in } \mathcal{P}\left(\mathbf{R}^{3} \times \mathbf{R}^{3}\right)
$$

uniformly in $t \in[0, T]$ as $N \rightarrow+\infty$; more precisely

$$
\sup _{t \in[0, T]} \operatorname{dist}_{M K R}\left(f_{\epsilon}(t, \cdot, \cdot), f_{N, \epsilon}(t, \cdot, \cdot)\right) \leq C(T, \epsilon) \operatorname{dist}_{M K R}\left(f^{i n}, f_{N}^{i n}\right)
$$

where $C(T, \epsilon)=(1+T L(T, \epsilon)) e^{T^{2} L(T, \epsilon)}$ with $L(T, \epsilon)$ as in (43). 
Proof. As we have explained before stating Theorem 5.1, the regularized Vlasov-Maxwell system (17) with initial data (18), or equivalently (21) with initial data (22) is a special case of (32) with integral operator $K_{\epsilon}$ defined by (41)-(42).

By Proposition 4.1, the system (32) with integral operator $K_{\epsilon}$ has a unique solution

$$
\left(X_{\epsilon}, \Xi_{\epsilon}\right) \equiv\left(X_{\epsilon}, \Xi_{\epsilon}\right)\left(t, x, \xi ; f^{i n}\right)
$$

for any initial Borel probability measure $f^{\text {in }}$ on $\mathbf{R}^{3} \times \mathbf{R}^{3}$.

On the other hand, if the map $t \mapsto\left(x_{i, N}(t), \xi_{i, N}(t)\right)_{1 \leq i \leq N}$ is $C^{1}$ on $\mathbf{R}_{+}$, a straightforward computation shows that it is a solution of (27) with initial data

$$
\left(x_{i, N}(0), \xi_{i, N}(0)\right)_{1 \leq i \leq N}=\left(x_{i, N}^{i n}, \xi_{i, N}^{i n}\right)_{1 \leq i \leq N}
$$

if and only if

$$
x_{i, N}(t)=X_{\epsilon}\left(t, x_{i, N}^{i n}, \xi_{i, N}^{i n} ; f_{N}^{i n}\right), \quad \xi_{i, N}(t)=\Xi_{\epsilon}\left(t, x_{i, N}^{i n}, \xi_{i, N}^{i n} ; f_{N}^{i n}\right) .
$$

This is in turn equivalent to the fact that the empirical measure

$$
f_{N, \epsilon}(t, \cdot, \cdot)=\frac{1}{N} \sum_{i=1}^{N} \delta_{x_{i, N}(t)} \otimes \delta_{\xi_{i, N}(t)}
$$

satisfies

$$
f_{N, \epsilon}(t, \cdot, \cdot)=\left(X_{\epsilon}, \Xi_{\epsilon}\right)\left(t, \cdot, \cdot ; f_{N}^{i n}\right) \# f_{N}^{i n} .
$$

Therefore the existence and uniqueness result in Proposition 4.1 translates into statement a) in the theorem.

Comparing this last equality with (40) and using the Dobrushin inequality in Proposition 4.3, we arrive at $\mathrm{b}$ ). (We recall that the distance dist $_{M K R}$ metrizes the topology of weak convergence on the set $\mathcal{P}\left(\mathbf{R}^{3} \times \mathbf{R}^{3}\right)$ of probability measures on $\mathbf{R}^{3} \times \mathbf{R}^{3}$.)

Theorem 5.1 can be viewed as a quantitative statement about the continuity in the weak topology of probability measures of the solution of the regularized Vlasov-Maxwell system (21) in terms of its initial data. Its corollary stated below explains how this information can be translated into uniform convergence of the $N$-particle regularized electromagnetic field to the one created by the solution of (21).

Let $\left(E_{\epsilon}, B_{\epsilon}\right)$ be the electromagnetic field associated to the solution of (21) with initial data $f^{i n}$ and let $\left(E_{N, \epsilon}, B_{N, \epsilon}\right)$ be the solution of the regularized Maxwell system

$$
\left\{\begin{array}{l}
\operatorname{div}_{x} B_{N, \epsilon}=0, \\
\partial_{t} B_{N, \epsilon}+\operatorname{curl}_{x} E_{N, \epsilon}=0, \\
\operatorname{div}_{x} E_{N, \epsilon}=\chi_{\epsilon} \star_{x} \chi_{\epsilon} \star_{x} \rho_{f_{N, \epsilon}}, \\
\partial_{t} E_{N, \epsilon}-\operatorname{curl}_{x} B_{N, \epsilon}=-\chi_{\epsilon} \star_{x} \chi_{\epsilon} \star_{x} j_{f_{N, \epsilon}},
\end{array}\right.
$$


with initial data

$$
\left.E_{N, \epsilon}\right|_{t=0}=-\nabla_{x} \chi_{\epsilon} \star_{x} \chi_{\epsilon} \star_{x} G \star_{x} \rho_{f_{N}^{i n}},\left.\quad B_{\epsilon}\right|_{t=0}=0
$$

In other words, $\left(E_{N, \epsilon}, B_{N, \epsilon}\right)$ is the regularized electromagnetic field created by the $N$-particle system.

Corollary 5.2. Under the same assumptions and with the same notations as in Theorem 5.1

a) for each $T>0$, one has

$$
\operatorname{dist}_{M K R}\left(\rho_{f_{\epsilon}}(t, \cdot), \rho_{f_{N, \epsilon}}(t, \cdot)\right) \leq C(T, \epsilon) \operatorname{dist}_{M K R}\left(f^{i n}, f_{N}^{i n}\right)
$$

and

$$
\left\|j_{f_{\epsilon}}(t, \cdot)-j_{f_{N, \epsilon}}(t, \cdot)\right\|_{W^{-1,1}\left(\mathbf{R}^{3}\right)} \leq 4 C(T, \epsilon) \operatorname{dist}_{M K R}\left(f^{i n}, f_{N}^{i n}\right)
$$

for all $t \in[0, T]$ and $N \geq 1$ - so that in particular

$$
\frac{1}{N} \sum_{i=1}^{N} \delta_{x_{i, N}(t)} \rightarrow \rho_{f_{\epsilon}}(t, \cdot) \quad \text { and } \quad \frac{1}{N} \sum_{i=1}^{N} v\left(\xi_{i, N}(t)\right) \delta_{x_{i, N}(t)} \rightarrow j_{f_{\epsilon}}(t, \cdot)
$$

uniformly in $t \in[0, T]$ as $N \rightarrow+\infty$, where the first convergence holds in the weak topology of $\mathcal{P}\left(\mathbf{R}^{3}\right)$, while the second holds in the weak topology of bounded Radon measures on $\mathbf{R}^{3}$;

b) for each $T>0,\left(E_{N, \epsilon}, B_{N, \epsilon}\right)$ converges to $\left(E_{\epsilon}, B_{\epsilon}\right)$ uniformly in $(t, x) \in[0, T] \times \mathbf{R}^{3}$ with the following estimates

$$
\left\|B_{\epsilon}-B_{N, \epsilon}\right\|_{L^{\infty}\left([0, T] \times \mathbf{R}^{3}\right)} \leq C^{\prime}(T, \epsilon) \operatorname{dist}_{M K R}\left(f^{i n}, f_{N}^{i n}\right)
$$

and

$$
\left\|E_{\epsilon}-E_{N, \epsilon}\right\|_{L^{\infty}\left([0, T] \times \mathbf{R}^{3}\right)} \leq C^{\prime \prime}(T, \epsilon) \operatorname{dist}_{M K R}\left(f^{i n}, f_{N}^{i n}\right),
$$

where

and

$$
C^{\prime}(T, \epsilon)=4 T C(T, \epsilon)\left\|\nabla_{x} Y_{\epsilon}\right\|_{L^{\infty}\left([0, T] ; W^{1, \infty}\left(\mathbf{R}^{3}\right)\right)}
$$

$$
\begin{array}{r}
C^{\prime \prime}(T, \epsilon)=4 T C(T, \epsilon)\left\|\nabla_{t, x} Y_{\epsilon}\right\|_{L^{\infty}\left([0, T] ; W^{1, \infty}\left(\mathbf{R}^{3}\right)\right)} \\
+\left\|d_{t} \nabla_{x} Y_{\epsilon} \star_{x} G\right\|_{L^{\infty}\left([0, T] ; W^{1, \infty}\left(\mathbf{R}^{3}\right)\right)},
\end{array}
$$

and where $C(T, \epsilon)$ is as in Theorem 5.1.

Proof. For the first statement in a), pick any bounded and Lipschitzcontinuous function $\phi$ on $\mathbf{R}^{3}$ and observe that

$$
\begin{array}{r}
\left|\int_{\mathbf{R}^{3}}\left(\rho_{f_{\epsilon}}(t, z)-\rho_{f_{N, \epsilon}}(t, z)\right) \phi(z) d x\right| \\
=\left|\iint_{\mathbf{R}^{3} \times \mathbf{R}^{3}}(\phi(x)-\phi(y)) \pi(t, d x d \xi d y d \eta)\right| \\
\leq 2\|\phi\|_{W^{1, \infty}} \iint_{\mathbf{R}^{3} \times \mathbf{R}^{3} \times \mathbf{R}^{3} \times \mathbf{R}^{3}} 1 \wedge|x-y| \pi(t, d x d \xi d y d \eta)
\end{array}
$$


where $\pi(t, \cdot)$ is any probability measure in $\Pi\left(f_{\epsilon}(t, \cdot), f_{N, \epsilon}(t, \cdot)\right)$. Minimizing the r.h.s. in $\pi(t, \cdot)$ while maximizing the l.h.s. in $\phi$ such that $\operatorname{Lip}(\phi) \leq 1$ and applying the Kantorovich duality theorem (see formula (5.11) on p. 60 in [19], or Theorem 1.3 on p. 19 in [18] and Remark 7.5 (i) on p. 207 in that same reference) leads to

$$
\operatorname{dist}_{M K R}\left(\rho_{f_{\epsilon}}(t, \cdot), \rho_{f_{N, \epsilon}}(t, \cdot)\right) \leq \operatorname{dist}_{M K R}\left(f_{\epsilon}(t, \cdot), f_{N, \epsilon}(t, \cdot)\right),
$$

and one concludes with Theorem $5.1 \mathrm{~b}$ ).

For the second statement, proceed in the same way, replacing the test function $\phi$ with $\psi(x) \cdot v(\xi)$, assuming that $\psi \in W^{1, \infty}\left(\mathbf{R}^{3} ; \mathbf{R}^{3}\right)$. Thus

$$
\begin{array}{r}
\left|\int_{\mathbf{R}^{3}}\left(j_{f_{\epsilon}}(t, z)-j_{f_{N, \epsilon}}(t, z)\right) \cdot \psi(z) d x\right| \\
=\left|\iint_{\mathbf{R}^{3} \times \mathbf{R}^{3}}(\psi(x) \cdot v(\xi)-\psi(y) \cdot v(\eta)) \pi(t, d x d \xi d y d \eta)\right| \\
\leq\left(2\|\phi\|_{W^{1, \infty}}+2\|\psi\|_{L^{\infty}}\right) \\
\times \iint_{\mathbf{R}^{3} \times \mathbf{R}^{3} \times \mathbf{R}^{3} \times \mathbf{R}^{3}}(1 \wedge|x-y|+1 \wedge|\xi-\eta|) \pi(t, d x d \xi d y d \eta) .
\end{array}
$$

Minimizing the r.h.s. in $\pi(t, \cdot)$ while maximizing the l.h.s. in $\psi$ such that $\|\psi\|_{W^{1, \infty}\left(\mathbf{R}^{3}\right)} \leq 1$ leads to

$$
\left\|j_{f_{\epsilon}}(t, \cdot)-j_{f_{N, \epsilon}}(t, \cdot)\right\|_{W^{-1,1}\left(\mathbf{R}^{3}\right)} \leq 4 \operatorname{dist}_{M K R}\left(f_{\epsilon}(t, \cdot), f_{N, \epsilon}(t, \cdot)\right)
$$

and one concludes again with Theorem $5.1 \mathrm{~b}$ ).

Now for b). Observe that

$$
B_{\epsilon}(t, x)-B_{N, \epsilon}(t, x)=\int_{0}^{t} \int_{\mathbf{R}^{3}} \nabla_{x} Y_{\epsilon}(t-s, x-y) \wedge\left(j_{f_{\epsilon}}(s, y)-j_{f_{N, \epsilon}}(s, y)\right) d y
$$

so that, for all $x \in \mathbf{R}^{3}$, one has

$$
\begin{aligned}
\left|B_{\epsilon}(t, x)-B_{N, \epsilon}(t, x)\right| \leq & t \sup _{0 \leq s \leq t}\left\|\nabla_{x} Y_{\epsilon}(s, \cdot)\right\|_{W^{1, \infty}\left(\mathbf{R}^{3}\right)} \\
& \times \sup _{0 \leq s \leq t}\left\|j_{f_{\epsilon}}(t, \cdot)-j_{f_{N, \epsilon}}(t, \cdot)\right\|_{W^{-1,1}\left(\mathbf{R}^{3}\right)}
\end{aligned}
$$


and one concludes by a). The estimate for the electric field is obtained in a similar way, from the formula

$$
\begin{aligned}
E_{\epsilon}(t, x) & -E_{N, \epsilon}(t, x) \\
& =-\int_{0}^{t} \int_{\mathbf{R}^{3}} \partial_{t} Y_{\epsilon}(t-s, x-y)\left(j_{f_{\epsilon}}(s, y)-j_{f_{N, \epsilon}}(s, y)\right) d y \\
& -\int_{0}^{t} \int_{\mathbf{R}^{3}} \nabla_{x} Y_{\epsilon}(t-s, x-y)\left(\rho_{f_{\epsilon}}(s, y)-\rho_{f_{N, \epsilon}}(s, y)\right) d y \\
& -\int_{\mathbf{R}^{3}}\left(\nabla_{x} \partial_{t} Y_{\epsilon}(t, \cdot) \star_{x} G\right)(x-y)\left(\rho_{f^{i n}}(y)-\rho_{f_{N}^{i n}}(y)\right) d y .
\end{aligned}
$$

\section{Final REMARKS AND CONCLUSiON}

The discussion above leaves aside several additional questions about this regularized dynamics.

First, although we insisted on choosing Rein's regularization procedure because the "pseudo-energy"

$$
\mathcal{W}\left[f_{\epsilon}\right](t):=\iint_{\mathbf{R}^{3} \times \mathbf{R}^{3}} e(\xi) f_{\epsilon}(t, x, \xi) d x d \xi+\int_{\mathbf{R}^{3}} \frac{1}{2}\left(\left|\tilde{E}_{\epsilon}\right|^{2}+\left|\tilde{B}_{\epsilon}\right|^{2}\right)(t, x) d x
$$

is constant under the dynamics of (21) - see Proposition 3.1 - we have not used this quantity in the mean-field limit itself. (The same can be said of earlier work in the same direction, first and foremost [3] and [7].)

Strictly speaking, Rein's theorem in [16] establishes that $\mathcal{W}\left[f_{\epsilon}\right]$ is constant only in the case where the initial particle distribution function $f^{i n}$ is a probability density (in fact, in the more general case where $f^{i n} \geq 0$ a.e. is a measurable function such that $\mathcal{W}\left[f^{i n}\right]<+\infty$.) Therefore, even though the empirical measure $f_{N, \epsilon}$ in Theorem 5.1 is a weak solution of the regularized Vlasov-Maxwell system in the sense of measures, Rein's theorem cannot be applied directly to initial data of the form $f_{N}^{i n}$.

Proposition 6.1. Let $N \geq 1$ and $\epsilon>0$ be fixed, let $\left(x_{i}^{i n}, \xi_{i}^{i n}\right)_{1 \leq i \leq N}$ be a $N$-tuple of elements in $\mathbf{R}^{3} \times \mathbf{R}^{3}$, and let

$$
f_{N}^{i n}:=\frac{1}{N} \sum_{i=1}^{N} \delta_{x_{i}^{i n}} \otimes \delta_{\xi_{i}^{i n}} .
$$


Let $t \mapsto\left(x_{i}(t), \xi_{i}(t)\right)_{1 \leq i \leq N}$ be the solution of (27) with initial data $\left(x_{i}^{i n}, \xi_{i}^{i n}\right)_{1 \leq i \leq N}$, and define

$$
f_{N, \epsilon}(t, \cdot, \cdot):=\frac{1}{N} \sum_{i=1}^{N} \delta_{x_{i}(t)} \otimes \delta_{\xi_{i}(t)}, \quad t \geq 0 .
$$

Then

$$
\mathcal{W}\left[f_{N, \epsilon}\right](t)=\mathcal{W}\left[f_{N}^{i n}\right]
$$

for all $t \geq 0$.

Proof. Regularize $f_{N}^{i n}$ in $(x, \xi)$, for instance by replacing $f_{N}^{i n}$ with its convolution $f_{N}^{i n} \star_{x, \xi} \zeta_{\eta}$ with any (nonnegative, compactly supported) mollifying sequence $\left(\zeta_{\eta}\right)_{\eta>0}$ on $\mathbf{R}^{3} \times \mathbf{R}^{3}$. If $\left|x_{i}^{i n}\right|,\left|\xi_{i}^{i n}\right|<R$ for each $i=1, \ldots, N$ and $\operatorname{supp}\left(\zeta_{\eta}\right) \subset B(0, \eta) \times B(0, \eta)$, one has

$$
\operatorname{supp}\left(f_{N, \eta}^{i n}\right) \subset B(0, R+\eta) \times B(0, R+\eta) .
$$

Then

$$
\tilde{E}_{N, \epsilon}^{i n}=-\nabla_{x} G \star_{x} \chi_{\epsilon} \star_{x} \rho_{f_{N}^{i n}} \text { and } \tilde{E}_{N, \eta, \epsilon}^{i n}=-\nabla_{x} G \star_{x} \chi_{\epsilon} \star_{x} \rho_{f_{N, \eta}^{i n}}
$$

both belong to $L^{2}\left(\mathbf{R}^{3}\right)$ since $\nabla_{x} G(x)=O\left(|x|^{-2}\right)$ as $|x| \rightarrow+\infty$.

Denote by $\left(f_{N, \eta, \epsilon}, E_{N, \eta, \epsilon}, B_{N, \eta, \epsilon}\right)$ the solution of (21) with initial data $\left(f_{N, \eta}^{i n}, \chi_{\epsilon} \star_{x} \tilde{E}_{N, \eta, \epsilon}^{i n}, 0\right)$. Applying Rein's theorem (Proposition 3.1) shows that

$$
\mathcal{W}\left[f_{N, \eta, \epsilon}\right](t)=\mathcal{W}\left[f_{N, \eta}^{i n}\right]<+\infty, \quad t \geq 0 .
$$

Let $\eta \rightarrow 0$. Then $f_{N, \eta}^{i n} \rightarrow f_{N}^{i n}$ in the weak topology of Borel probability measures on $\mathbf{R}^{3}$, and

$$
\iint_{\mathbf{R}^{3} \times \mathbf{R}^{3}} e(\xi) f_{N, \eta}^{i n}(x, \xi) d x d \xi \rightarrow \iint_{\mathbf{R}^{3} \times \mathbf{R}^{3}} e(\xi) f_{N}^{i n}(d x d \xi)
$$

since $\operatorname{supp}\left(f_{N, \eta}^{i n}\right) \subset B(0, R+\eta) \times B(0, R+\eta)$. On the other hand, denoting

$$
\theta_{\eta}(x):=\int_{\mathbf{R}^{3}} \zeta_{\eta}(x, \xi) d \xi,
$$

which is a regularizing sequence on $\mathbf{R}^{3}$, we see that

$$
\tilde{E}_{N, \eta, \epsilon}^{i n}=\theta_{\eta} \star_{x} \tilde{E}_{N, \epsilon}^{i n} \rightarrow \tilde{E}_{N, \epsilon}^{i n}
$$

in $L^{2}\left(\mathbf{R}^{3}\right)$. Therefore

$$
\mathcal{W}\left[f_{N, \eta}^{i n}\right] \rightarrow \mathcal{W}\left[f_{N}^{i n}\right]<+\infty \quad \text { as } \eta \rightarrow 0 .
$$

Reasoning as in the proof of Theorem 5.1 and Corollary 5.1, since $f_{N, \eta, \epsilon}$ and $f_{N, \epsilon}$ are two solutions of the same regularized Vlasov-Maxwell 
system with initial data satisfying $f_{N, \eta}^{i n} \rightarrow f_{N}^{i n}$ in weak- $\mathcal{P}\left(\mathbf{R}^{3} \times \mathbf{R}^{3}\right)$ as $\eta \rightarrow 0$, we deduce from Dobrushin's estimate that

$$
f_{N, \eta, \epsilon}(t, \cdot, \cdot) \rightarrow f_{N, \epsilon}(t, \cdot, \cdot) \text { in weak- } \mathcal{P}\left(\mathbf{R}^{3} \times \mathbf{R}^{3}\right)
$$

uniformly in $t \in[0, T]$ for each $T>0$, while

$$
\left(\tilde{E}_{N, \eta, \epsilon}, \tilde{B}_{N, \eta, \epsilon}\right) \rightarrow\left(\tilde{E}_{N, \epsilon}, \tilde{B}_{N, \epsilon}\right)
$$

uniformly on $[0, T] \times \mathbf{R}^{3}$ for each $T>0$ as $\eta \rightarrow 0$. (Recall that $\left(\tilde{E}_{N, \eta, \epsilon}, \tilde{B}_{N, \eta, \epsilon}\right)$ is the solution of the regularized Maxwell system (19) with initial data $(20)$ with $f_{N, \eta, \epsilon}$ in the place of $f_{\epsilon}$, while $\left(\tilde{E}_{N, \epsilon}, \tilde{B}_{N, \epsilon}\right)$ is the solution of the same system with $f_{N, \epsilon}$ in the place of $f_{\epsilon}$.)

Obviously $\operatorname{supp}\left(f_{N, \eta, \epsilon}(t, \cdot, \cdot)\right) \subset B(0, R+\eta+t) \times \mathbf{R}^{3}$ (since particles travel at speed $<1)$. Since

$$
\int_{\mathbf{R}^{3}} \rho_{f_{N, \eta, \epsilon}}(t, x) d x=1 \text { and } \int_{\mathbf{R}^{3}}\left|j_{f_{N, \eta, \epsilon}}(t, x)\right| d x \leq 1
$$

we conclude from the formulas expressing $E_{N, \eta, \epsilon}$ and $B_{N, \eta, \epsilon}$, i.e.

$$
\begin{aligned}
& E_{N, \eta, \epsilon}=-\partial_{t} \nabla_{x} Y_{\epsilon} \star_{x} G \star_{x} \rho_{f_{N, \eta}^{i n}}-\nabla_{x} Y_{\epsilon} \star_{t, x} \rho_{f_{N, \eta, \epsilon}}-\partial_{t} Y_{\epsilon} \star_{t, x} j_{f_{N, \eta, \epsilon}} \\
& B_{N, \eta, \epsilon}=\operatorname{curl}_{x}\left(Y_{\epsilon} \star_{t, x} j_{f_{N, \eta, \epsilon}}\right)
\end{aligned}
$$

that there exists $C_{\epsilon, T}>0$ such that

$$
\left|E_{N, \eta, \epsilon}(t, x)\right|+\left|B_{N, \eta, \epsilon}(t, x)\right| \leq C_{\epsilon, T}
$$

uniformly in $\eta>0$ as $(t, x) \in[0, T] \times \mathbf{R}^{3}$. Therefore, for each $t \in[0, T]$, one has

$$
\operatorname{supp}\left(f_{N, \eta, \epsilon}(t, \cdot, \cdot)\right) \subset B(0, R+\eta+T) \times B\left(0, R+\eta+2 T C_{\epsilon, T}\right)
$$

uniformly in $\eta>0$, and

$$
\operatorname{supp}\left(\rho_{f_{N, \eta, \epsilon}}(t, \cdot)\right), \operatorname{supp}\left(j_{f_{N, \eta, \epsilon}}(t, \cdot)\right) \subset B(0, R+\eta+t) .
$$

We also deduce from the formulas expressing $\tilde{E}_{N, \eta, \epsilon}$ and $\tilde{B}_{N, \eta, \epsilon}$ that, if $0 \leq t \leq T$, then

$$
\tilde{E}_{N, \eta, \epsilon}-\partial_{t} Y \star_{x} \tilde{E}_{N, \eta, \epsilon}^{i n} \text { and } \tilde{B}_{N, \eta, \epsilon}
$$

have compact support in $[0, T] \times B(0, R+\eta+\epsilon+2 T)$.

Since we already know that $E_{N, \eta, \epsilon}^{i n} \rightarrow E_{N, \epsilon}^{i n}$ in $L^{2}\left(\mathbf{R}^{3}\right)$ and that $\left(\tilde{E}_{N, \eta, \epsilon}, \tilde{B}_{N, \eta, \epsilon}\right) \rightarrow\left(\tilde{E}_{N, \epsilon}, \tilde{B}_{N, \epsilon}\right)$ uniformly on $[0, T] \times \mathbf{R}^{3}$ for each $T>0$ as $\eta \rightarrow 0$, we conclude that

$$
\mathcal{W}\left[f_{N, \eta, \epsilon}\right](t) \rightarrow \mathcal{W}\left[f_{N, \epsilon}\right](t)
$$

for each $t \geq 0$ as $\eta \rightarrow 0$, so that

$$
\mathcal{W}\left[f_{N, \epsilon}\right](t)=\mathcal{W}\left[f_{N, \epsilon}^{i n}\right]
$$

for all $t \geq 0$. 
Since the quantity $\mathcal{W}\left[f_{N, \epsilon}\right](t)$ is an invariant of the motion for the dynamics of (27), a natural question would be to check whether (27) is a hamiltonian system. We have left this question unanswered for lack of applications of immediate interest. Besides, since the mollified dynamics (27) is not a fundamental law of physics, whether it is hamiltonian is perhaps a purely academic question.

Several remarks are in order to conclude the analysis presented above.

First, we have chosen to study (27) instead of (28) because empirical measures constructed on the trajectories of (27) are exact solutions of (21) in the measure sense. This interpretation disappears if one neglects the self-interaction term in the regularized system. However, because of Proposition 3.2, both regularized systems are equivalent for $\epsilon>0$ fixed and $N \rightarrow+\infty$. On the contrary, none of these systems seem to make very much sense for $\epsilon=0$, and in any case modeling self-interaction in classical electrodynamics is a source of conceptual difficulties, as mentioned above. As explained on p. 677 in [8], there is little hope of deriving the Vlasov-Maxwell system as a mean-field limit of a particle system without regularization as long as self-interaction is not better understood.

Also, the work presented here leaves aside several issues mentioned in [8] without further analysis, such as including spin in the particle system; as in [8], the regularization procedure used here (based on convolution with a blob function in the space variable only) is not Lorentz invariant. In any case, this regularization should not be viewed as an attempt to depart from a strict point particle model and introduce some physical effect involving particle size, but as a mere mathematical expedient.

As a matter of fact, the regularized dynamics (27) could be regarded as an analogue for the Vlasov-Maxwell system of the well-known vortex blob method used in numerical simulations of incompressible fluid flows: see section 5.3 in [14] for a concise, yet lucid presentation of this method. In the present context, one has the following approximation result.

Proposition 6.2. Let $f^{\text {in }} \in L^{\infty}\left(\mathbf{R}^{3} \times \mathbf{R}^{3}\right)$ be a compactly supported probability density on $\mathbf{R}^{3} \times \mathbf{R}^{3}$.

For each $N \geq 1$, let $\left(x_{i, N}^{i n}, \xi_{i, N}^{i n}\right)_{1 \leq i \leq N} \in\left(\mathbf{R}^{3} \times \mathbf{R}^{3}\right)^{N}$ be such that

$$
\frac{1}{N} \sum_{i=1}^{N} \delta_{x_{i, N}^{i n}} \otimes \delta_{\xi_{i, N}^{i n}} \rightarrow f^{i n} d x d \xi \text { weakly in } \mathcal{P}\left(\mathbf{R}^{3} \times \mathbf{R}^{3}\right)
$$

in the limit as $N \rightarrow+\infty$. 
For each $\epsilon>0$, let $t \mapsto\left(x(t)_{i, N, \epsilon}, \xi(t)_{i, N, \epsilon}\right)_{1 \leq i \leq N}$ be the solution of the regularized dynamics (27). Then, there exists subsequences $N_{k} \rightarrow+\infty$ and $\epsilon_{k} \rightarrow 0$ such that

$$
\frac{1}{N} \sum_{i=1}^{N} \delta_{x_{i, N_{k}, \epsilon_{k}}(t)} \otimes \delta_{\xi_{i, N_{k}, \epsilon_{k}}(t)} \rightarrow f(t, \cdot, \cdot) d x d \xi \text { weakly in } \mathcal{P}\left(\mathbf{R}^{3} \times \mathbf{R}^{3}\right)
$$

uniformly in $t \in[0, T]$ for each $T>0$, where $(f, E, B)$ is a global weak solution of the Vlasov-Maxwell system (1) with initial data (2)-(3).

The global existence of weak solutions of the Vlasov-Maxwell system was obtained by DiPerna-Lions [6], with some additional precisions to be found in [16]. The (very weak) approximation result above follows from applying Rein's Proposition 4 in [16] and Theorem 5.1. Indeed, let $f_{\epsilon}$ be the solution of the regularized Vlasov-Maxwell system (17) with initial data (18) for each $\epsilon>0$.

By Proposition 4 in [16], there exists a subsequence $\epsilon_{k} \rightarrow 0$ such that $f_{\epsilon_{k}}(t, \cdot, \cdot) d x d \xi \rightarrow f(t, \cdot, \cdot) d x d \xi$ in weak- $\mathcal{P}\left(\mathbf{R}^{3} \times \mathbf{R}^{3}\right)$ uniformly in $t \in[0, T]$ for all $T>0$ as $\epsilon_{k} \rightarrow 0$. Therefore, for each $k>0$ and $T>0$, there exists $\epsilon_{k}>0$ small enough so that

$$
\operatorname{dist}_{M K R}\left(f(t, \cdot, \cdot), f_{\epsilon_{k}}(t, \cdot, \cdot)\right) \leq 2^{-k-1} \text { for each } t \in[0, T] \text {. }
$$

Then, for this value of $\epsilon_{k}>0$, by Theorem 5.1, there exists $N_{k}>0$ large enough so that

$$
\operatorname{dist}_{M K R}\left(f_{\epsilon_{k}}(t, \cdot, \cdot), f_{N_{k}, \epsilon_{k}}(t, \cdot, \cdot)\right) \leq 2^{-k-1} \text { for each } t \in[0, T],
$$

where

$$
f_{N_{k}, \epsilon_{k}}(t, \cdot, \cdot):=\frac{1}{N} \sum_{i=1}^{N} \delta_{x_{i, N_{k}, \epsilon_{k}}(t)} \otimes \delta_{\xi_{i, N_{k}, \epsilon_{k}}(t)} .
$$

One then concludes by the triangle inequality.

Finally, it could be interesting to study fluctuations as Braun and Hepp [3] did for the Vlasov-Poisson case; this is left for future investigation.

Acknowledgements. The author is indebted to V. Ricci for discussions about this problem and the one analyzed in [8]. Part of this work was completed during a visit to Kyoto University and it is a pleasure to thank Prof. K. Aoki and the Department of Mechanical Engineering and Science for their kind hospitality and support.

\section{REFERENCES}

[1] F. Bouchut, F. Golse, C. Pallard: Classical solutions and the Glassey-Strauss theorem for the 3D Vlasov-Maxwell system, Archive for Rational Mech. and Anal. 170 (2003), 1-15. 
[2] F. Bouchut, F. Golse, C. Pallard: Nonresonant smoothing for coupled wave+transport equations and the Vlasov-Maxwell system, Rev. Mat. Iberoamericana 20 (2004), 865-892.

[3] W. Braun, K. Hepp: The Vlasov dynamics and its fluctuations in the $1 / n$ limit of interacting classical particles, Comm. in Math. Phys. 56 (1977), 101-113.

[4] S. Calogero, G. Rein: On classical solutions of the NordströmVlasov system, Comm. Partial Differential Equations 28 (2003), 1863-1885.

[5] Y. Choquet-Bruhat: Problème de Cauchy pour le système intégro-différentiel d'Einstein-Liouville, Ann. Inst. Fourier 21 (1971), 181-201.

[6] R.J. DiPerna, P.-L. Lions: Global weak solutions of Vlasov-Maxwell systems, Comm. on Pure and Appl. Math. 42 (1989), 729-757.

[7] R. L. Dobrushin: Vlasov equations; Func. Anal. Appl. 13 (1979), 115-123.

[8] Y. Elskens, M. K.-H. Kiessling, V. Ricci: The Vlasov limit for a system of particles which interact with a wave field, Comm. in Math. Phys. 285 (2009), 673-712.

[9] L. Erdös, H.T. Yau: Derivation of the nonlinear Schrödinger equation from a many body Coulomb system, Adv. Theor. Math. Phys. 5 (2001), 1169-1205.

[10] M. Hauray, P.-E. Jabin: N-particle approximation of the Vlasov equations with singular potential, Arch. Rational Mech. Anal. 183 (2007), 489-524.

[11] J.D. Jackson: "Classical Electrodynamics". John Wiley and Sons Inc. 1999.

[12] M. K.-H. Kiessling: Microscopic derivatons of Vlasov equations, Comm. Nonlin. Sci. Numer. Simul. 13 (2008), 106-113.

[13] A.A Luchina, A.A. Vlasov: Chapter II, section 12, of [21].

[14] C. Marchioro, M. Pulvirenti: "Mathematical Theory of Incompressible Nonviscous Fluids", Springer-Verlag, New York 1994.

[15] H. Neunzert, J. Wick: Die Approximation der Lösung von IntegroDifferentialgleichungen durch endliche Punktmengen; Lecture Notes in Math. vol. 395, 275-290, Springer, Berlin (1974).

[16] G. Rein: Global weak solutions of the relativistic Vlasov-Maxwell system revisited, Comm. in Math. Sci. 2 (2004), 145-158.

[17] H. Spohn: "Dynamics of Charged Particles and their Radiation Fields". Cambridge Univ. Press, Cambridge 2004.

[18] C. Villani: "Topics in Optimal Transportation". American Math. Soc. Providence RI 2003.

[19] C. Villani: "Optimal Transport: Old and New". Springer-Verlag, BerlinHeidelberg 2009.

[20] A.A. Vlasov: On Vibration Properties of Electron Gas (in Russian), J. Exp. Theor. Phys. 8 (3) (1938), 291.

[21] A.A. Vlasov: "Many-Particle Theory and Its Application to Plasma", Gordon and Breach, New York 1961.

(F. G.) Ecole polytechnique, Centre de mathématiques L. Schwartz, F91128 Palaiseau Cedex, \& Université Paris-Diderot, Laboratoire J.-L.

LiOns, BP 187, F75252 PARIS CEDEX 05

E-mail address: francois.golse@math.polytechnique.fr 